\title{
An Accurate Wall Temperature Measurement Using Infrared Thermometry with Enhanced Two-Phase Flow Visualization in a Convective Boiling System
}

\author{
Junsoo Yoo ${ }^{\mathrm{a},{ }^{*}}$, Carlos E. Estrada-Perez ${ }^{\mathrm{b}}$, Yassin A. Hassan ${ }^{\mathrm{a}, \mathrm{b}}$ \\ ${ }^{a}$ Department of Nuclear Engineering, Texas A\&M University. 337 Zachry Engineering Building, 3133 TAMU, College Station, \\ Texas 77843-3133, USA \\ ${ }^{b}$ Department of Mechanical Engineering, Texas A\&M University, 100 MEOB, 3123 TAMU, College Station, Texas 77843-3123, \\ USA \\ *Corresponding author. Tel.: +1 979-845-4161; Email address: kaks2000@tamu.edu (J. Yoo)
}

\begin{abstract}
This paper presents an experimental strategy to achieve the accurate wall temperature measurement using infrared (IR) thermometry with the enhanced flow visualization. Our particular interest is focused on the measurement of two-phase flow parameters in a convective boiling system which involves a large heated area. For the present application, the important issues such as the design of test section and material selection were discussed along with our decision-making process. Then, the IR-based temperature tracking algorithm was established based on the multi-layer heater wall design proposed. To apply this algorithm, however, the optical properties of materials must be identified first. Thus, the optical features of selected materials, e.g., soda-lime glass and indium-tin-oxide (ITO) were investigated and the measured values were validated through experiments.

The wall temperature tracking algorithm on the proposed heater wall design was validated both for steadystate and transient conditions. Also, such algorithm was proved to be applicable for the heat flux measurement. Finally, the feasibility of the present approach was demonstrated through a subcooled flow boiling experiment. The results showed that both the hydrodynamic motions of bubble and the corresponding wall temperature can be captured with high fidelity using the measurement strategy presented, from which the interrelation between the sliding vapor bubbles and the wall heat transfer were discussed.
\end{abstract}

Key words: Infrared thermometry, flow visualization, subcooled boiling, Indium-Tin-Oxide film, soda-lime glass 


\section{Introduction}

The heat transfer augmentation caused by boiling has been of great concern to researchers due to its potential to improve the cooling systems of many engineering applications. Often, the performance of engineering devices such as electronics and nuclear reactors has been limited by the amount of power that can be dissipated while using them because the operating temperature must be kept within specified limits to maintain the reliability. However, the traditional cooling systems employing the single-phase forced convection have limitations in addressing this issue, implying that alternative approach like boiling heat transfer needs to be introduced to make a breakthrough.

In order to realize the enhanced heat transfer of boiling in engineering applications, the physical mechanism should be understood well so that we can predict its performance with reasonable accuracy. In view of this, numerous experimental efforts have been made to obtain better insight into the boiling mechanism with various experimental techniques. Among such efforts, optical methods such as flow visualization and infrared (IR) thermometry are considered effective because they can provide direct observation of the thermal-hydraulic features related to boiling. Consequently, high-speed photography (Klausner et al., 1993; Situ et al., 2008; Chu et al., 2011) and a laser-based flow visualization technique (Estrada-Perez and Hassan, 2010) have been utilized to study the hydrodynamics of liquid/vapor phases in a boiling system. For a wall heat transfer study, the thermal patterns under boiling conditions can be also visualized, for which liquid crystal thermography (Kenning et al., 2001; Bayazit et al., 2003; Muwanga and Hassan, 2006) and infrared (IR) thermometry (Hapke et al., 2000; Theofanous et al., 2002a; Theofanous et al., 2002b; Diaz et al., 2006; Jung et al., 2014) have been employed.

The above-mentioned optical techniques have an advantage over traditional methods relying on local probe sensors because the hydrodynamic and thermal features of interest can be captured non-intrusively with high spatial and temporal resolution. As a result, even the micro-physics of the two-phase flow such as wall boiling and local mass/momentum/energy transfer can be characterized without any disturbance of instrument. Also, using a measurement of macro-scale, the transitional changes between different flow regimes, e.g., single-phase/two-phase flow regions, bubbly/slug/annular flow regimes, can be captured over a large area.

In recent years, rapidly growing computing power and advances in high-speed imaging technology have led to aspirations among researchers to combine high-speed IR thermometry with the high-speed visualization techniques. So far, such attempts have been made primarily in pool boiling experiments (Golobic et al., 2009; 
Gerardi et al., 2010; Kunkelmann et al., 2012) with a few flow boiling experiments in mini-/micro-channels (Barber et al., 2009). All of these experiments were designed to observe the bubble dynamics and the wall temperature field simultaneously. This is obviously an appealing experimental approach because it can provide direct insight into the relation between hydrodynamic bubble motions and wall heat transfer. However, such efforts are rare in general convective boiling experiments, especially for technical applications involving a large heated area.

To achieve reliable data measurement from both high-speed flow visualization and IR thermometry, several sources of measurement error, especially optical issues, must be addressed correctly. Otherwise, the reliability of the parameters measured will decline substantially, despite the fancy-looking visual images obtained from such methods. Therefore, to implement both techniques in a single experimental facility, several issues must be taken into particular consideration. First, the materials composing the test section should be determined by considering their optical properties; and the experimental design should consider the feasibility both for flow visualization and IR thermometry. Also, since both techniques provide optical images from which quantifiable data can be acquired, optical distortions should be prevented. Additionally, to accurately estimate the target object's temperature from the thermal images taken by IR camera, proper calibration considering the optical property is required.

In this paper, we discuss the experimental strategy assuring both enhanced flow visualization and accurate wall temperature measurement using high-speed photography and IR thermometry. Our particular interest is focused on the improved observation of two-phase flow hydrodynamics as well as the wall temperature distribution in a convective boiling system which involves a large heated area. For this approach, the heater wall design is especially important because it directly affects the quality of IR temperature measurement and the flow visualization. In literatures, about three different types of heater wall configurations have been utilized for the boiling study employing IR thermometry. Some of those are described here with brief descriptions of the wall temperature measurement strategies, after which the relevance of each approach for the present application is discussed.

\section{(i) A heating channel coated with IR-opaque material}

A heating channel lined with IR-opaque material of high emissivity has been primarily used in flow boiling experiments in mini- and micro-channels. Hapke et al. (2000) examined the wall heat transfer characteristics under flow boiling conditions in a mini-channel. They painted the outer wall of the test section with black lacquer (emissivity $\approx 0.94$ ) to ensure high sensitivity of the IR temperature measurement. Then, the outer-wall temperature 
distribution was measured by IR camera, from which the inner wall temperature touching the fluid was evaluated by assuming quasi-steady conditions of the wall. The similar approach was also taken by Boye et al. (2007) who performed a flow boiling experiment with water in a circular mini-channel. On the other hand, Barber et al. (2009) attempted the high-speed photography along with IR thermometry in a rectangular micro-channel made of borosilicate glass. In order to visualize the boiling motions inside the channel while measuring the outer wall temperature using IR camera, they employed the transparent metallic deposit on the outer wall of the glass channel. Then, the local heat transfer characteristics at different flow boiling regimes were studied.

\section{(ii) A thin metal foil heater}

Recently, to observe the wall heat transfer characteristics during boiling, a thin metal foil on the order of micrometers thickness has been used as a heater by itself. This approach has been adopted to perform fundamental boiling studies with high spatial and temporal resolution at the local nucleation site. Golobic et al. (2009) performed an experiment under subcooled and saturated pool boiling conditions with water at atmospheric pressure. To create the boiling, they used a platinum heating foil of $6 \mu \mathrm{m}$ thickness with a $2-3 \mu \mathrm{m}$ thick layer of black paint on the back side. Then, a high-speed IR camera measured the two-dimensional wall temperature field during the growth of bubbles. Schweizer and Stephan (2009) studied the nucleate boiling of FC-72 under variable gravitational conditions. The measurements were performed at a single artificial cavity created on a thin heating foil made of stainless steel $25 \mu \mathrm{m}$ thick. Similarly to Golobic et al. (2009), Schweizer and Stephan (2009) applied a coat of black paint to the back side of the heating foil for the IR thermometry. Kunkelmann et al. (2012) investigated the effect of three-phase contact line motion on the local evaporative heat transfer within a micro-region. For the heater in this experiment, a stainless steel foil $20 \mu \mathrm{m}$ thick was used, and the wall temperature was measured using IR camera while bubbles' motions were recorded by high-speed video camera.

\section{(iii) A heating film deposited on an IR-transparent substrate}

The heater wall temperature can be also measured using IR camera through an IR-transparent substrate onto which an IR-opaque heating film is deposited. Theofanous et al. (2002b) used an IR camera to visualize the dynamic thermal patterns on the heated wall in a pool boiling experiment. As a heating element, titanium films of 140-1000 nm thickness were deposited on $130 \mu \mathrm{m}$ thick borosilicate glass. Then, the IR camera captured the 
thermal footprint created by the boiling motion through the glass substrate. In Gerardi et al. (2010), a transparent and electrically conductive indium-tin-oxide (ITO) film was used as a heating element for their saturated pool boiling study. The IR-opaque ITO film was attached to a $0.4 \mathrm{~mm}$ thick sapphire substrate through which the bubble nucleation and the transient wall temperature were observed. Recently, in Fischer et al. (2012), an enhanced emissivity of chromium-based layer was applied on a $2 \mathrm{~mm}$ thick IR-transparent calcium fluoride $\left(\mathrm{CaF}_{2}\right) \mathrm{substrate}$ and a pure chromium heating layer was laid on top of that. Then, an IR camera detected the wall temperature through the $\mathrm{CaF}_{2}$ substrate during the boiling process. In Kim et al. (2012), a silicon wafer was employed as a substrate of the heater wall, and a polyimide tape coated with a thin black paint was attached to the substrate to improve the signal detected by IR camera for their wall heat transfer study.

\section{(iv) Discussion on the previous experimental design approaches}

The relevance of each approach described above for the present application can be discussed based on the specific measurement targets of the present study. The first approach (i) is inapplicable because the flow visualization is restricted by the opaque film on the outer wall of the test channel. Even in the case that the channel wall and the heating element are configured for enabling visualization as in Barber et al. (2009), the IR camera cannot directly measure the surface temperature of the inner wall touching the fluid due to the IR-opaque film on the outer wall. Thus, the time-varying inner wall temperature due to the boiling is hard to capture with this approach unless the channel wall is extremely thin. The second approach (ii), which employs a thin metal foil as a heater, is better for capturing the transient wall temperature induced by boiling using the IR camera. However, achieving a large heated area is difficult because of the structurally weak nature of the thin foil. Also, the low heat capacity of thin foil can alter the boiling process compared to the thicker heaters. Therefore, in the present study we based the heater wall design on the third approach (iii). However, we avoided using the well-known IR transparent materials such as $\mathrm{CaF}_{2}$, sapphire, and zinc selenide as a substrate because they are usually very expensive and/or problematic to treat as a large piece. Instead, soda-lime glass was used, for which the additional effort for calibration is necessary to accurately measure the wall temperature.

A series of extensive work required to achieve the high-fidelity measurement employing both the IR thermometry and the high-speed photography are described in this paper. As shown in Figure 1, the present efforts were made by considering the general process that must be pursued for phasing in the new experimental techniques 
or approaches properly. We treated all the details, including the test section design, IR measurement issues, visualization strategy, wall temperature tracking method, and experimental validations. Then, the subcooled flow boiling experiment was performed using the verified approach, during which we could clearly see the detailed relation between the bubble motions and the wall heat transfer as the bubbles develop throughout the flow channel.

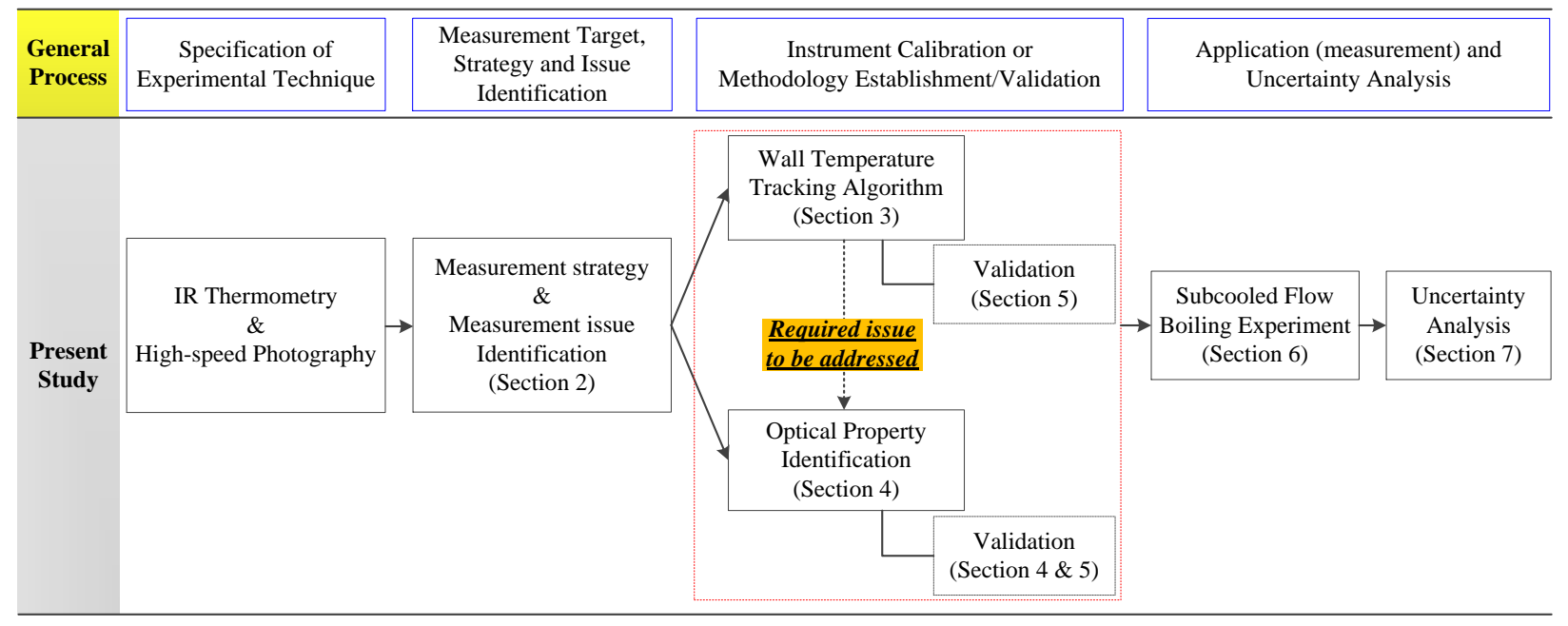

Figure 1. Overview of present study considering the general process for introducing the new experimental approach

2. Experimental strategy and measurement issues

\subsection{Material selection and test section design}

For the simultaneous application of high-speed photography and IR thermometry, the test section walls should be transparent in visible light; whereas, the heater surface (i.e., wall) touching the fluid should be opaque to IR radiation so that the wall temperature can be measured by IR camera. If a thin heating film is deposited on the substrate, the substrate should be at least semi-transparent to the IR spectral range of interest so that the varying temperature on the film can be directly captured through the substrate using IR camera. A high-speed IR camera (SC8000, FLIR Systems, Inc.) working for a mid-wave IR (i.e., 3-5 $\mu \mathrm{m}$ ) range was utilized in this study.

The mechanical strength of substrate is also an important factor to consider because we want to investigate the flow boiling features over a relatively large heated area. Also, the thermal properties of substrate are important because they can affect the temperature profile within the substrate which subsequently affects the determination of the local heat transfer parameters (Fischer et al., 2012; Kim et al., 2012). For instance, high thermal conductivity 
would readily smear out any temperature gradients within the substrate, resulting in a lower magnitude of temperature difference across the substrate used to estimate the local heat transfer coefficient or local heat flux. Accordingly, the test section for the flow boiling experiment was designed as shown in Figure 2. The channel geometry is square $\left(10 \times 10 \mathrm{~mm}^{2}\right)$, and the fluid flows upward through a vertical test section with a total height of $305 \mathrm{~mm}$ and a heated length $\left(\mathrm{L}_{0}\right)$ of $265 \mathrm{~mm}$ on one side. The test section is enclosed by transparent acrylic walls except for the heater wall side. On the heater wall side, ITO film is attached to a soda-lime glass substrate. The ITO film is exposed to the test fluid, $3 \mathrm{M}^{\mathrm{TM}}$ Novec $^{\mathrm{TM}} 7000$, within the channel and is heated by an electrical current.

As shown in Figure 2 (top), the heater wall has multi-layer configuration which is to improve the insulating features of test section. In particular, a sapphire window is installed next to the glass substrate so that the trapped air between the glass substrate and sapphire window can prevent the heat loss to the environment. Note that all the materials composing the test section, including the ITO film, are transparent to visible light. Thus, the illuminating light for flow visualization is available from every direction around the test section (Figure 2, bottom), which ensures enhanced bubble imaging with high-speed video cameras. Meanwhile, since ITO film is opaque to midwave IR radiation while the soda-lime glass substrate and sapphire window are semi-transparent to such IR range, the ITO temperature can be measured through the multilayer walls. The soda-lime glass was utilized as substrate for the ITO film due to its availability and cost effectiveness compared to well-known IR-transparent materials as well as its thermal, optical, and mechanical properties. Specifically, soda-lime glass has low thermal conductivity $(\approx 0.94$ $\mathrm{W} / \mathrm{m}-\mathrm{K}$ ), is semi-transparent to mid-wave IR radiation, and is mechanically strong enough to be treated at a large size. However, soda-lime glass has not been widely used in IR thermometry, and the specific composition may change depending on the manufacturer. Therefore, in order to achieve the accurate wall temperature measurement, the properties of such materials, especially the optical properties, should be verified first (Section 4). Also, the thickness of glass substrate should be proper to capture the reasonable amount of IR radiation originating from the ITO. Here, we used $0.5 \mathrm{~mm}$ thick soda-lime glass (Bayview Optics, Inc.). 


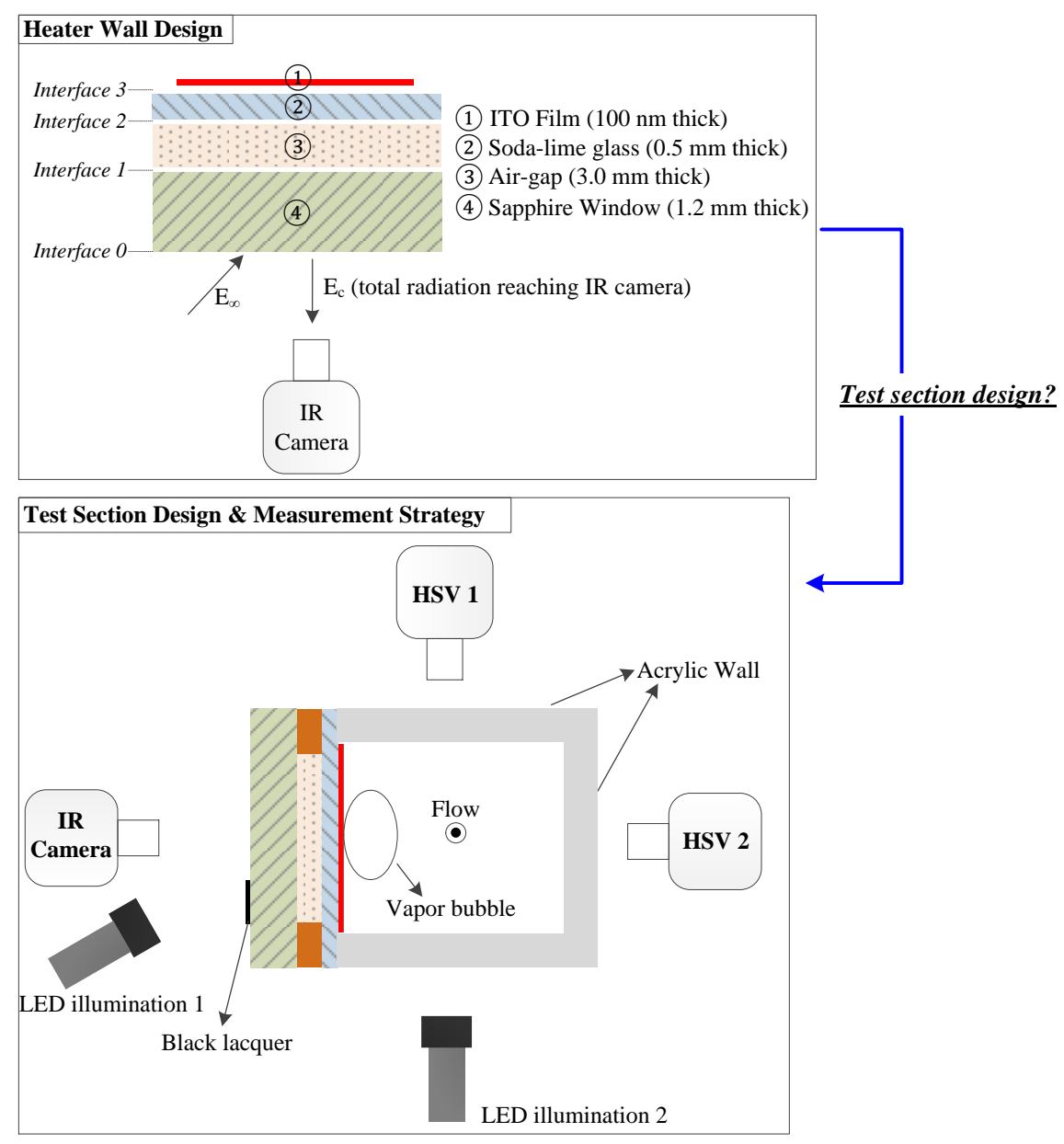

Figure 2. Heater wall design (top) and measurement strategy (bottom) in the subcooled flow boiling experiment (view from the top of channel)

\subsection{Measurement strategy}

In Figure 2 (bottom), the cameras arrangement applied to the present subcooled flow boiling experiment is shown. Two high-speed video cameras were used to observe vapor bubble motions while the temperature of the wall (i.e., ITO film) is measured by an IR camera located on the heater wall side. To improve the image quality taken from both high-speed video cameras, LED illumination was used from two sides. Also, the two high-speed video cameras (HSV 1 and HSV 2) are synchronized so that the bubbles' behavior can be observed from different views. On the outer surface of the sapphire window, black lacquer was painted on one edge to provide the boundary condition for the wall temperature tracking (see Section 3). This experimental setup allows capturing the detailed boiling motions along with the corresponding wall temperature field. 


\subsection{Wall temperature measurement issues}

The ITO has been often employed in previous boiling studies aiming to visualize the boiling motions along with the wall temperature measurement using IR thermometry due to its unique optical feature (Okawa et al., 2005; Gerardi et al., 2010; Trujillo et al., 2011). However, we should note that substantial errors might be caused in IRbased temperature measurement unless special attention is paid due to the ITO's high reflectivity of IR radiation.

Figure 3 shows the axial wall temperature profile (total length $\mathrm{L}_{0}=265 \mathrm{~mm}$ ) measured by IR camera for the unheated (left) and heated (right) single-phase upward flow. The axial temperature profile is expected to be flat in the unheated case and linear in the heated case, but the obvious temperature distortions are found in Figure 3 . This is because the IR radiation from the heated body of IR camera was reflected onto the surface of the ITO film, and the reflected radiation returned back to the IR camera, which led to the distortion in the temperature measurement of the wall (ITO film). This phenomenon is called the Narcissus effect (Horny, 2003; Vollmer and Mollmann, 2011). The camera lens and/or the instruments around the test section can create similar reflective effects unless the temperature measurement is performed with special care. To prevent such reflection, we used a black rubber plate to cover the front of the IR camera except for the lens. In addition, the IR camera was positioned at a small angle to the heater surface instead of being perpendicular, which was to avoid the reflection caused by the optical lens.

Due to the low emissivity of ITO $(\varepsilon \approx 0.16$, see section 4 and 5), surrounding conditions such as ambient temperature may also have substantial impacts on the IR-based temperature measurement in the present application. Therefore, the related uncertainty should be carefully addressed (Sections 5 and 7). Furthermore, due to the multilayer design of heater wall (Figure 2), the IR camera cannot directly capture the correct wall temperature because the total energy measured by the IR camera $\left(E_{c}\right)$ consists of the energy emitted by the several layers composing the heater wall. Thus, this multi-layer wall effect should be taken into consideration, which is discussed in the following section. 

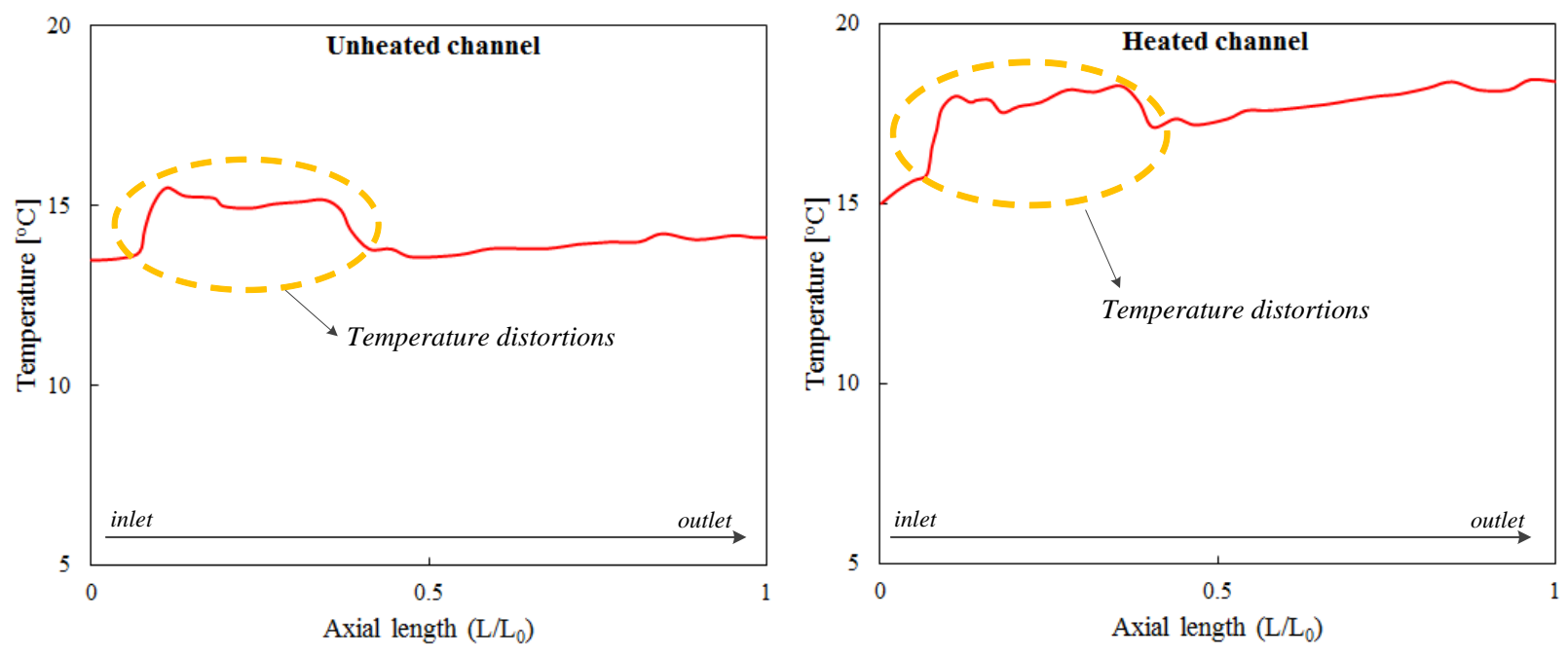

Figure 3. Temperature distortions captured by the IR camera due to the Narcissus effect

3. Analysis method for wall temperature tracking

In Figure 2, we can easily find that the IR radiation originating from the ITO film would decrease significantly as it goes through the glass substrate and sapphire window before reaching the IR camera. This implies that the amount of IR radiation emitted by the ITO film must be estimated correctly for accurate wall temperature measurement. Here, the total radiation reaching the IR camera consists of energy emitted by several layers, i.e., ITO film, soda-lime glass, and sapphire, leading to the following radiation balance equation:

$$
\mathrm{E}_{\mathrm{c}}=\rho_{\infty-\mathrm{c}} \mathrm{E}_{\infty}+\varepsilon_{\text {sap }-\mathrm{c}} \mathrm{E}_{\text {sap }}+\varepsilon_{\mathrm{g}-\mathrm{c}} \mathrm{E}_{\mathrm{g}}+\tau_{\text {ITO- } \mathrm{c}} \mathrm{E}_{\text {ITO }}
$$

where $E_{c}$ is the total radiation measured by the IR camera; $E_{\infty}$ is the background radiation introduced by the surroundings $\left(\mathrm{E}_{\infty}=\sigma \mathrm{F}_{3-5 \mu \mathrm{m}}(\mathrm{T}) \mathrm{T}_{\infty}^{4}\right) ; E_{\text {sap }}, E_{g}$, and $E_{I T O}$ are the emission from the sapphire, soda-lime glass, and ITO film, respectively; $\sigma$ is the Stefan-Boltzmann constant $\left(\mathrm{W} / \mathrm{m}^{2}-\mathrm{K}^{4}\right)$; and $T_{\infty}$ is the ambient temperature. $\mathrm{F}_{3-5 \mu \mathrm{m}}$ represents the fraction of radiation energy within the wavelength interval 3-5 $\mu \mathrm{m}$, which can be obtained from tables or by numerically integrating Planck's distribution (Incropera and Dewitt, 2002). Note that the radiation emitted by air is neglected in Eq. (1), because the transmissivity of air can be assumed as unity (i.e., $\tau_{\infty}=1$ ) at short distances between the IR camera and the target object, implying that the emission/reflection due to air can be neglected.

The coefficients of each term used in Eq. (1) were determined based on the approach employed by Kim et al. (2012). Following their approach, the infinite number of transmissions, absorptions, and reflections of radiation 
across the media composing the heater wall is considered to obtain the analytical expressions of those coefficients. The derivations are described in detail in Appendix A.3. The final forms of each coefficient are given as follows:

$$
\begin{aligned}
& \rho_{\infty-c}=\rho_{\text {sap }-\infty}+\frac{\left(1-\rho_{\text {sap }-\infty}\right)^{2} \tau_{\text {sap }}^{2} \rho_{\text {app, sap }-\infty, 1}}{1-\rho_{\text {sap }-\infty} \rho_{\text {app }, \text { sap }-\infty, 1} \tau_{\text {sap }}^{2}} \\
& \varepsilon_{\text {sap-c }}=\frac{\left(1-\rho_{\text {sap }-\infty}\right)\left(1+\rho_{\text {app, sap }-\infty, 1} \tau_{\text {sap }}\right)}{1-\rho_{\text {sap }-\infty} \rho_{\text {app }, \text { sap }-\infty, 1} \tau_{\text {sap }}^{2}} \\
& \varepsilon_{\mathrm{g}-\mathrm{c}}=\left[\frac{\left(1-\rho_{\mathrm{g}-\infty}\right)\left(1+\rho_{\text {ITO-g }} \tau_{\mathrm{g}}\right)}{1-\rho_{\mathrm{g}-\infty} \rho_{\text {ITO-g }} \tau_{\mathrm{g}}^{2}}\right]\left[\frac{1-\rho_{\text {sap }-\infty}}{1-\rho_{\text {sap }-\infty} \rho_{\text {app }, \mathrm{g}-\infty, 2}}\right]\left[\frac{\left(1-\rho_{\text {sap }-\infty}\right) \tau_{\text {sap }}}{1-\rho_{\text {sap }-\infty} \rho_{\text {app }, \text { sap }-\infty, 1} \tau_{\text {sap }}^{2}}\right] \\
& \tau_{\text {ITO-c }}=\left[\frac{\tau_{\mathrm{g}}}{1-\rho_{\mathrm{g}-\infty} \rho_{\text {ITO-g }} \tau_{\mathrm{g}}}\right]\left[\frac{1-\rho_{\mathrm{g}-\infty}}{1-\rho_{\text {sap }-\infty} \rho_{\text {app }, \mathrm{g}-\infty, 2}}\right]\left[\frac{\left(1-\rho_{\text {sap }-\infty}\right)^{2} \tau_{\text {sap }}}{1-\rho_{\text {sap }-\infty} \rho_{\text {app }, \text { sap }-\infty, 1} \tau_{\text {sap }}^{2}}\right]
\end{aligned}
$$

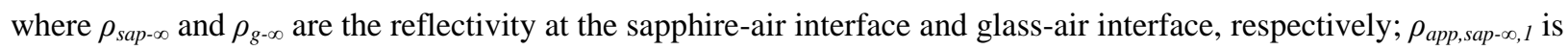
the apparent reflectivity at interface 1 between the sapphire and the air gap; $\rho_{a p p, g-\infty, 2}$ is the apparent reflectivity at interface 2 between the glass and the air gap; $\rho_{\text {IтO-g }}$ is the reflectivity at interface 3 between the ITO film and the glass; and $\tau_{g}$ and $\tau_{s a p}$ are the transmissivity of glass and sapphire, respectively. Note that, for the semi-transparent medium to IR radiation (e.g., glass, sapphire), the concepts of apparent reflectivity $\left(\rho_{a p p, m-\infty}\right)$ and apparent transmissivity $\left(\tau_{a p p, m-\infty}\right)$ are used in addition to the normal reflectivity $\left(\rho_{m-\infty}\right)$ and normal transmissivity $\left(\tau_{m}\right)$, which is to consider the infinite number of reflections/transmissions throughout the medium (see Appendix A.1).

Due to the high absorptivity/emissivity of IR radiation of soda-lime glass, the temperature profile across the medium can substantially affect the amount of radiation reaching the IR camera. Thus, we employed the 1D heat conduction equation to obtain the temperature profile across the multi-layer, which is coupled with Eq. (1) as in Kim et al. (2012). The overall algorithm is described as follows.

Given the assumed initial temperature profile across the multi-layer, ambient temperature $\left(T_{\infty}\right)$, and total radiation captured by the IR camera $\left(E_{c}\right)$, the wall temperature $\left(T_{\text {ITO }}\right)$ is obtained first using Eqs. (1). The wall temperature obtained is then used as one of the boundary conditions for the subsequent transient heat conduction simulation. The other boundary condition is given by the temperature measured by the IR camera on the outer surface of the sapphire window where the black lacquer is, as shown in Figure 2. Then, by solving the heat conduction equation with these boundary conditions, the temperature profile across the multi-layer wall is updated. Subsequently, the wall temperature $T_{i t o}$ at the new time step can be obtained using Eqs. (1) in the same way 
mentioned before. Repeating this process allows the tracking of time-dependent wall temperature variation. It is noted that as the initial temperature profile across the multi-layer is arbitrarily assumed, the true temperature of the wall can be obtained after the influence of initial condition is dampened out. Kim et al. (2012) proved that this twoway coupled algorithm works in the multi-layer design of wall composed of silicon substrate, polyimide tape, and black paint. However, such method has not been attempted and/or validated yet for the materials adopted in our study for which the optical properties must be identified.

\section{Optical property measurement}

\subsection{Experimental procedure and measured optical properties}

The optical properties, i.e., reflectivity, transmissivity, and emissivity of the materials were measured using an experimental setup shown in Figure 4 with a blackbody source (Infrared Systems Development Corp., emissivity $\left.\varepsilon_{B . B}=0.96 \pm 0.02\right)$. In this study, we were particularly concerned with how the properties of the selected materials, in particular the ITO and soda-lime glass, behave within the temperature and spectral ranges of interest, which were $20-85^{\circ} \mathrm{C}$ and 3-5 $\mu \mathrm{m}$. In general, optical properties depend on temperature and wavelength; and such dependency may affect the performance of the present wall temperature tracking discussed in Section 3. Therefore, to measure the optical properties of the target objects and to evaluate the temperature dependency of such properties, an airtemperature-control system was installed as shown in Figure 4. Using this system, the temperature of target object was controlled by injection of cold or hot air; and the temperature measurement using IR camera was conducted at different temperatures of target object without any disturbance during the measurement because the transmissivity of air is always almost unity. 
(i) Test for reflectivity

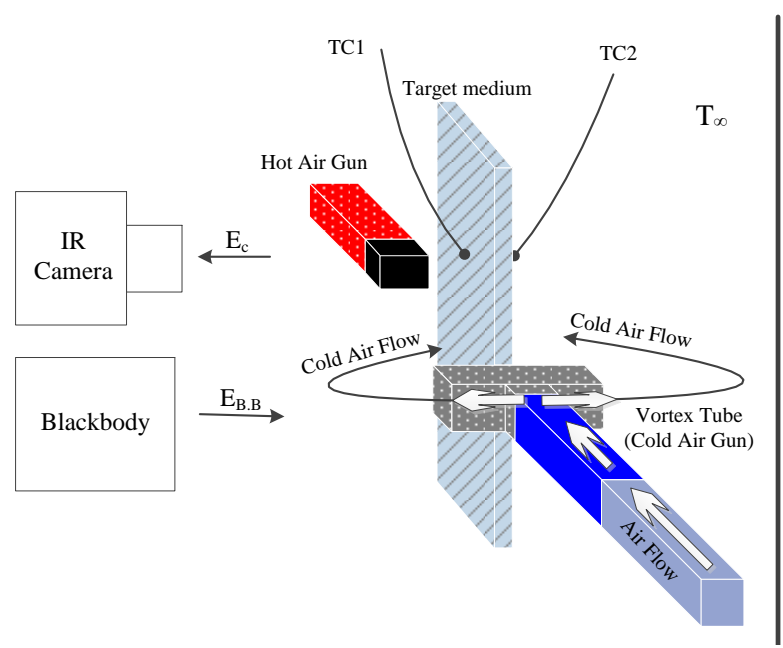

(ii) Test for transmissivity

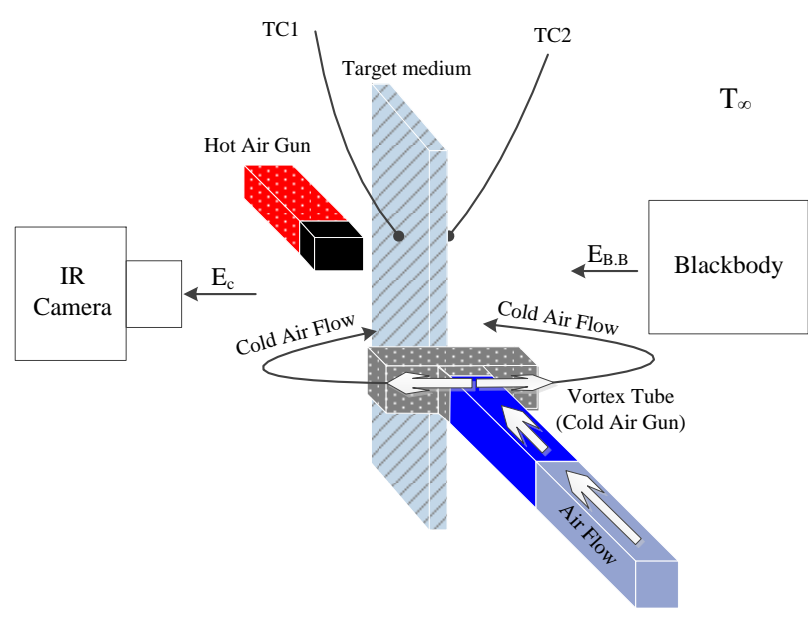

Figure 4. Optical property measurement of the materials composing the heater wall

The specific procedures for optical property measurement are as follows. (1) A blackbody source temperature is fixed at a certain level $\left(60^{\circ} \mathrm{C}\right)$. (2) The thermocouple tips (K-type) are placed at symmetrical locations on each side of the medium within the area in which the temperature, or more accurately the radiation, is measured by the IR camera (see Figure 4). (3) Hot or cold air is injected toward the target medium until the surface temperature of both sides of the medium reaches a steady state as measured by the thermocouples. The injection of hot or cold air must be carefully controlled to achieve surface temperatures as similar as possible on both sides of the medium because this allows the simple assumption of a uniform temperature profile across the medium. (4) At each temperature of the target medium, the total radiation $\left(E_{c}\right)$ is measured using the IR camera with and without a blackbody source. Then, using the difference between the total radiation with and without a blackbody source, the reflectivity and transmissivity can be obtained. Note that the total radiation reaching the IR camera is determined by the (i) emission, (ii) reflection, and (iii) transmission of radiation throughout the medium:

$$
\mathrm{E}_{\mathrm{c}}=\mathrm{E}_{\text {emission }}+\mathrm{E}_{\text {reflection }}+\mathrm{E}_{\text {transmission }}
$$

where $E_{\text {emission }}$ is the radiation emitted by the medium $\left(=\varepsilon_{a p p, m-\infty} F_{3-5 \mu m}(T) \sigma T_{m}{ }^{4}\right) ; E_{\text {reflection }}$ is the radiation reflected by the medium; and $E_{\text {transmission }}$ is the radiation transmitted through the medium.

In the experimental setup shown in Figure 4 (left side), $E_{\text {reflection }}$ is determined by the blackbody source temperature $\left(=\rho_{a p p, m-\infty} F_{3-5 \mu m}(T) \sigma \varepsilon_{B . B} T_{B . B}{ }^{4}\right)$ or the ambient temperature $\left(=\rho_{a p p, m-\infty} F_{3-5 \mu m}(T) \sigma T_{\infty}{ }^{4}\right)$, depending on whether a blackbody source is used or not. In contrast, neither $E_{\text {emission }}$ nor $E_{\text {transmission }}$ depends on the existence of a blackbody source as long as $T_{m}$ and $T_{\infty}$ are fixed. Therefore, using the difference of total radiation captured by the IR 
camera $\left(E_{c}\right)$ between the measurement with and without a blackbody source, the $\rho_{a p p, m-\infty}$ at a certain $T_{m}$ can be obtained based on the following equation:

$$
\begin{aligned}
& \left(\mathrm{E}_{\mathrm{c}}\right)_{\text {with B.B }}-\left(\mathrm{E}_{\mathrm{c}}\right)_{\text {without B.B }} \\
& =\left(\mathrm{E}_{\text {emission }}+\mathrm{E}_{\text {reflection }}+\mathrm{E}_{\text {transmission }}\right)_{\text {with B.B }}-\left(\mathrm{E}_{\text {emission }}+\mathrm{E}_{\text {reflection }}+\mathrm{E}_{\text {transmission }}\right)_{\text {without B.B }} \\
& =\left(\mathrm{E}_{\text {reflection }}\right)_{\text {with B.B }}-\left(\mathrm{E}_{\text {reflection }}\right)_{\text {without B.B }} \\
& =\rho_{\text {app }, \mathrm{m}-\infty} \mathrm{F}_{3-5 \mu \mathrm{m}}(\mathrm{T}) \sigma \varepsilon_{\mathrm{B} . \mathrm{B}} \mathrm{T}_{\mathrm{B} . \mathrm{B}}^{4}-\rho_{\text {app }, \mathrm{m}-\infty} \mathrm{F}_{3-5 \mu \mathrm{m}}(\mathrm{T}) \sigma \mathrm{T}_{\infty}^{4}
\end{aligned}
$$

In the case of a reflectivity measurement for the ITO film using Eq. (7), the normal reflectivity ( $\left.\rho_{\text {ITO }}\right)$ is used instead of apparent reflectivity because the ITO film is assumed to be completely opaque to IR radiation, implying that transmission and reflection within the film do not exist. In other words, the radiation phenomenon is treated as a surface phenomenon instead of a volumetric phenomenon for opaque materials like ITO.

The $\tau_{a p p, m-\infty}$ was measured based on the experimental setup shown in Figure 4 (right side). Here, the $E_{\text {transmission }}$ depends on the blackbody source temperature $\left(=\tau_{a p p, m-\infty} F_{3-5 \mu m}(T) \sigma \varepsilon_{B . B} T_{B . B}{ }^{4}\right)$ or the ambient temperature $(=$ $\tau_{a p p, m-\infty} F_{3-5 \mu m}(T) \sigma T_{\infty}^{4}$ ), depending on whether a blackbody source is used or not. Meanwhile, since neither $E_{\text {emission }}$ nor $E_{\text {reflection }}$ is affected by the use of a blackbody source while $T_{m}$ and $T_{\infty}$ are kept same, $\tau_{\mathrm{app}, \mathrm{m}-\infty}$ can be obtained based on the following equation:

$$
\begin{aligned}
& \left(\mathrm{E}_{\mathrm{c}}\right)_{\text {with B.B }}-\left(\mathrm{E}_{\mathrm{c}}\right)_{\text {without B.B }} \\
& =\left(\mathrm{E}_{\text {emission }}+\mathrm{E}_{\text {reflection }}+\mathrm{E}_{\text {transmission }}\right)_{\text {with B.B }}-\left(\mathrm{E}_{\text {emission }}+\mathrm{E}_{\text {reflection }}+\mathrm{E}_{\text {transmission }}\right)_{\text {without B.B }} \\
& =\left(\mathrm{E}_{\text {transmission }}\right)_{\text {with B.B }}-\left(\mathrm{E}_{\text {transmission }}\right)_{\text {without B.B }} \\
& =\tau_{\mathrm{app}, \mathrm{m}-\infty} \mathrm{F}_{3-5 \mu \mathrm{m}}(\mathrm{T}) \sigma \varepsilon_{\mathrm{B} . \mathrm{B}} \mathrm{T}_{\mathrm{B} . \mathrm{B}}^{4}-\tau_{\mathrm{app}, \mathrm{m}-\infty} \mathrm{F}_{3-5 \mu \mathrm{m}}(\mathrm{T}) \sigma \mathrm{T}_{\infty}^{4}
\end{aligned}
$$

In addition to optical property measurements for different temperatures of the target medium, we also measured transmissivity of the soda-lime glass while varying the blackbody source temperature over the range $40-80^{\circ} \mathrm{C}$.

Table 1 shows the measured properties of the materials, i.e., the ITO film, soda-lime glass and sapphire. The major sources of uncertainty during the measurements were (i) temperature fluctuations of the target medium during the air cooling/heating process, (ii) the local temperature gradient within the measurement area, and (iii) the emissivity of the blackbody source. The effects of (i) and (ii) increased when the temperatures of the target medium were over $60^{\circ} \mathrm{C}$. Nevertheless, the measured properties did not show noticeable changes under the various test conditions applied during this study. The uncertainty of the measured optical properties shown in Table 1 was obtained by taking the twice of standard deviation of the repeated measurements at various test conditions to ensure a $95 \%$ confidence level. 
Once $\rho_{a p p, m-\infty}$ and $\tau_{a p p, m-\infty}$ are determined for each target object, $\rho_{m-\infty}$ and $\tau_{m}$ can be obtained using the following relationships:

$$
\begin{aligned}
\rho_{\mathrm{app}, \mathrm{m}-\infty} & =\rho_{\mathrm{m}-\infty}+\frac{\left(1-\rho_{\mathrm{m}-\infty}\right)^{2} \rho_{\mathrm{m}-\infty} \tau_{\mathrm{m}}^{2}}{1-\rho_{\mathrm{m}-\infty}^{2} \tau_{\mathrm{m}}^{2}} \\
\tau_{\mathrm{app}, \mathrm{m}-\infty} & =\frac{\left(1-\rho_{\mathrm{m}-\infty}\right)^{2} \tau_{\mathrm{m}}}{1-\rho_{\mathrm{m}-\infty}^{2} \tau_{\mathrm{m}}^{2}}
\end{aligned}
$$

Because Eqs. (9) and (10) are non-linear systems of equations dependent on each other, the solutions can be found through an iterative process based on the measured properties (i.e., $\left.\rho_{a p p, m-\infty}, \tau_{a p p, m-\infty}\right)$ until the unknowns (i.e., $\left.\rho_{m-\infty}, \tau_{m}\right)$ are found. Equations (9) and (10) are derived in Appendix A.1; the derivation can also be found in Kim et al. (2012). After obtaining $\rho_{m-\infty}$ and $\tau_{m}$, the absorption coefficient $(a)$ can be estimated based on the relationship $\tau=\exp (-a L)$ derived from Beer's law. In addition, the refractive index, or the reflectivity at the interfaces (e.g., $\rho_{\text {ITO-g }}$, see Appendix A.3.), can be estimated using the Fresnel equation, which is widely used to represent radiation behavior across an interface between two media. Assuming that the incoming radiation is perpendicular to the flat surface of target medium, the Fresnel equation is given by

$$
\rho_{\mathrm{A}-\mathrm{B}}=\left(\frac{\mathrm{n}_{\mathrm{A}}-\mathrm{n}_{\mathrm{B}}}{\mathrm{n}_{\mathrm{A}}+\mathrm{n}_{\mathrm{B}}}\right)^{2}
$$

where $\rho_{A-B}$ is the reflectivity at the interface between the media $\mathrm{A}$ and $\mathrm{B}$, and $n$ is the refractive index. When the medium comes in contact with air $(\infty), \rho_{A-\infty}$ can be obtained if $n_{A}$ is known while $n_{A}$ can be obtained if $\rho_{A-\infty}$ is known by assuming $n_{\infty}=1$. In this way, all the properties required to solve the Eq. (1) can be defined.

The refractive index of the soda-lime glass and the sapphire used in this study were estimated based on the measured properties (Table 1) and using Eqs. (9) through (11). Then, for validation purposes, the refractive index values obtained were compared with those found in the literature. The refractive index of sapphire $\left(n_{\text {sap }}\right)$ is known to be about $1.6 \sim 1.7$ at $\lambda=3-5 \mu \mathrm{m}$ while the estimated value based on the present measurements was $n_{\text {sap }} \approx 1.63$. In the case of the soda-lime glass, finding the refractive index $\left(n_{g}\right)$ within the spectral range of interest was difficult; but $n_{g}$ is known to be about 1.5 at $\lambda=1-2 \mu \mathrm{m}$ while the refractive index estimated based on the measured properties in Table 1 was $\mathrm{n}_{\mathrm{g}} \approx 1.41$. These results indicate that the measured values are reasonable. To confirm the validity of these measured properties for the temperature tracking, further validation was performed and is discussed in Section 4.2. 
Table 1. Measured optical properties of the materials composing the heater wall

\begin{tabular}{|c|c|c|}
\hline & Nominal Value & Uncertainty \\
\hline Apparent transmissivity of glass $\left(\tau_{a p p, g-\infty}\right)$ & 0.42 & 0.018 \\
\hline Apparent reflectivity of glass $\left(\rho_{a p p, g-\infty}\right)$ & 0.04 & 0.004 \\
\hline Reflectivity of ITO $\left(\rho_{\text {ITO }}\right)$ & 0.82 & 0.019 \\
\hline Apparent reflectivity of sapphire $\left(\rho_{a p p, s a p-\infty}\right)$ & 0.11 & 0.002 \\
\hline 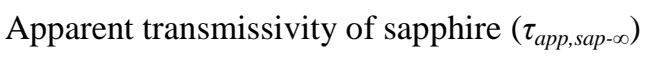 & 0.84 & 0.018 \\
\hline
\end{tabular}

\subsection{Validation of measured properties of semi-transparent media}

To validate the measured properties of soda-lime glass and sapphire, several tests were performed. Specifically, the IR camera, blackbody source, and the semi-transparent medium employed in this study were arranged similarly to the experimental setup for the transmissivity measurement (Figure 4, right side). Then, the blackbody source temperature was observed through the semi-transparent medium using the IR camera. The radiation balance equation and the derivation of coefficients required for these tests are given in Appendix A.2. $T_{\infty}$ was measured by a T-type thermocouple, and the semi-transparent medium was assumed to stay in thermal equilibrium with the surroundings, meaning that temperature variation within the medium is ignored.

Firstly, the measurements were performed with a single layer of soda-lime glass and with a single layer of sapphire window placed between the IR camera and the blackbody source (i.e., single-layer test). The radiation emitted by the blackbody source was measured through the single layer of each medium, and calibration was performed using the measured optical properties (Table 1) as well as the corresponding radiation balance equation (see Appendix A.2). The results are shown in Figure 5, which shows that after applying the calibration, the measured (or tracked) blackbody source temperature agrees well with that measured by the thermocouple, within 0.7 ${ }^{\circ} \mathrm{C}$ for the soda-lime glass and $0.4^{\circ} \mathrm{C}$ for the sapphire. The assumptions of thermal equilibrium and a uniform temperature profile across the medium are considered the major sources of uncertainty during this test. For the sodalime glass, the assumptions seem to have more impact on the temperature tracking results than for the sapphire due to the high emissivity of the soda-lime glass. Figure 5 also shows the difference between the direct temperature measurements using the IR camera without calibration and the ones when calibrated. Note that the simple adjustment of the emissivity value in the IR camera software (ExaminIR, FLIR Systems Inc.) is not enough to make 
this temperature measurement accurate, because the volumetric effect of the radiation phenomenon within the semitransparent medium cannot be accounted for in that way.
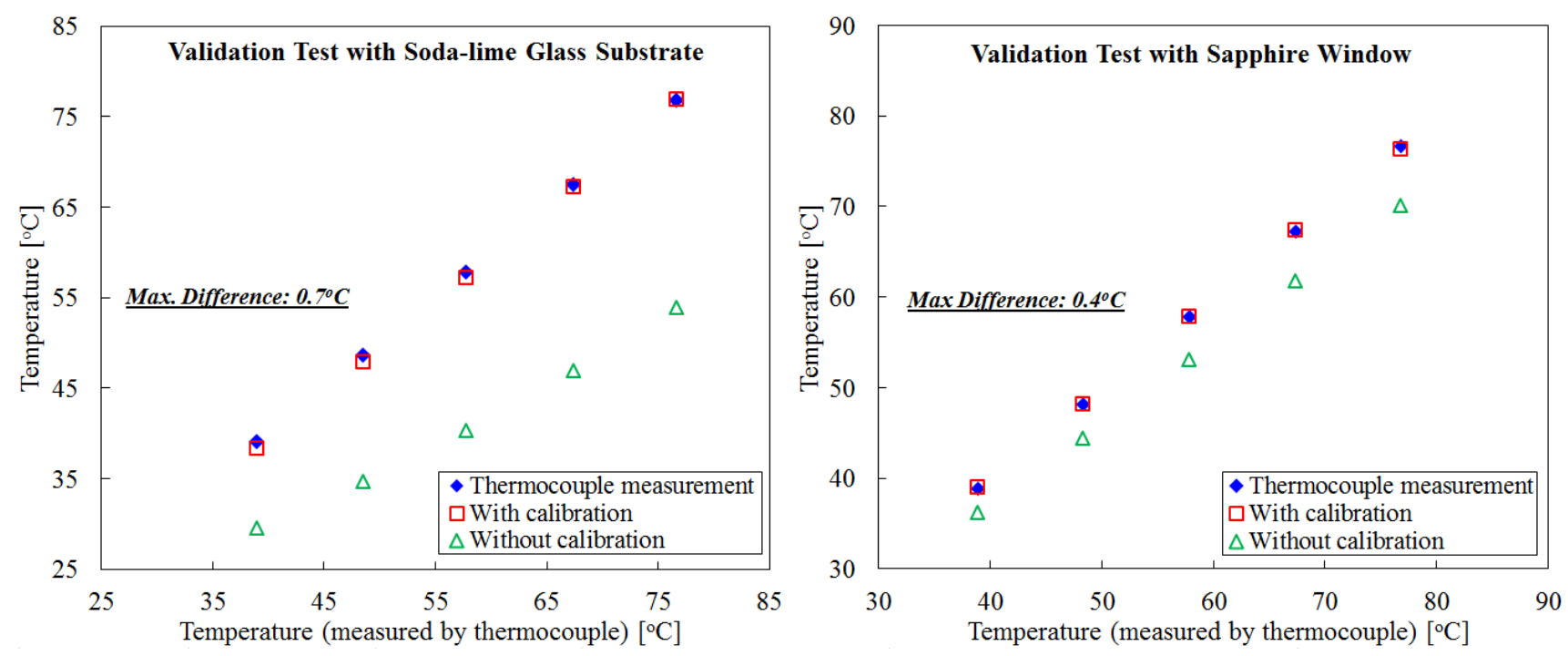

Figure 5. Temperature tracking results through a single layer of a semi-transparent medium

After a test using a single medium, the sapphire window and soda-lime glass were placed together between the IR camera and the blackbody source so that the blackbody source temperature could be measured through both media (i.e., double-layer test). The radiation balance equation and the derivation of coefficients for this test are given in Appendix A.2. Figure 6 shows the temperature tracking results for the double-layer test; the maximum deviation compared to the direct measurements using thermocouple was found to be $0.6{ }^{\circ} \mathrm{C}$. The main sources of uncertainty during this test are considered to be the same as those for the single-layer test. From the results shown in Figures 5 and 6, we concluded that the measured optical properties of the soda-lime glass and the sapphire window shown in Table 1 are valid enough for accurate temperature tracking through these media using IR thermometry. 


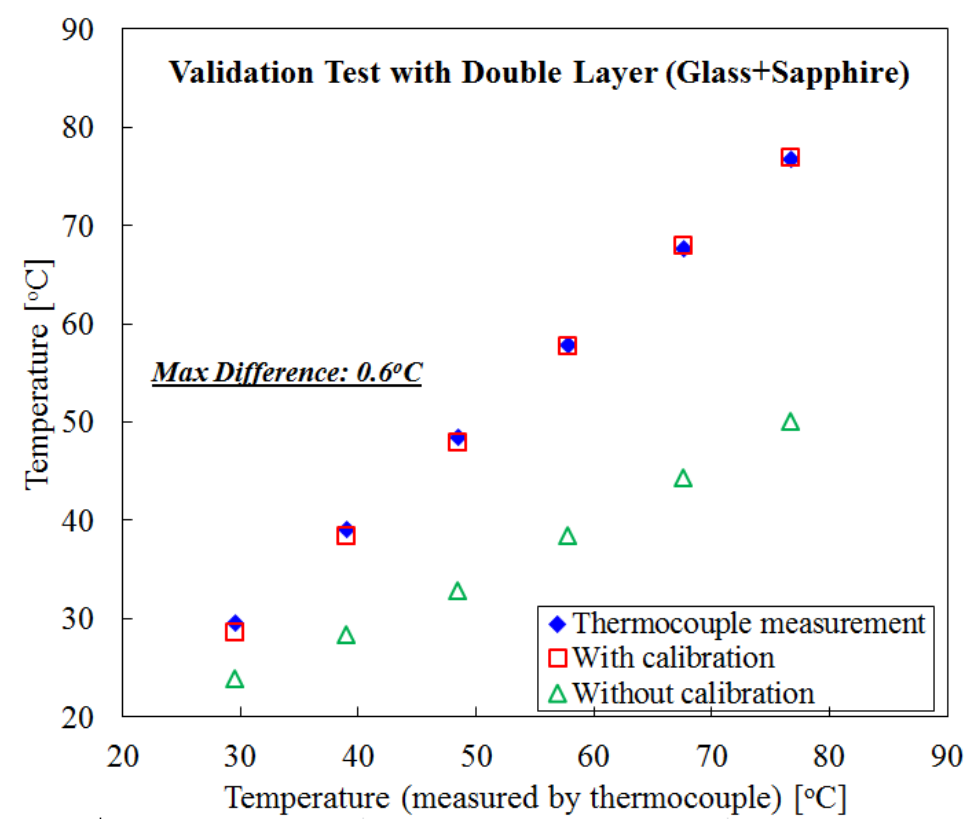

Figure 6. Temperature tracking results through the double layer of semi-transparent media

5. Experimental validation with the proposed heater wall design

The multi-layer design of heater wall shown in Figure 2 contains a thin ITO film attached to the soda-lime glass substrate. Thus, the optical properties of the ITO should be verified as well. Also, the overall performance of the wall temperature tracking algorithm should be demonstrated before it is applied to the actual application, e.g., subcooled flow boiling experiment, which are described in this section.

5.1. Validation of ITO film's optical properties and the steady-state test

Due to the opaqueness of ITO to IR radiation (i.e., $\left.\tau_{I T O}=0\right)$, the emissivity of ITO $\left(\varepsilon_{\text {ITO }}\right)$ can be determined based on the measured reflectivity shown in Table 1 along with the following relation:

$$
\alpha_{\text {гто }}+\rho_{\text {Іто }}=\varepsilon_{\text {Іто }}+\rho_{\text {Іто }}=1
$$

where $\alpha$ is the absorptivity, $\rho$ is the reflectivity, $\varepsilon$ is the emissivity, and $\alpha=\varepsilon$ is assumed according to Kirchhoff's law.

When treating low emissivity and IR-opaque material such as ITO, the optical properties must be determined with special care, otherwise small errors in the measured properties may cause substantial errors in the final result of temperature tracking. Therefore, to confirm the validity of ITO's measured property, we performed the validation test for which the similar experimental setup shown in Kim et al. (2012) was utilized which is 
presented in Figure 7. During this test, the same heater wall design shown in Figure 2 was used, and the ITO temperature was observed from both sides simultaneously using gold-coated mirrors which are highly reflective of IR radiation ( $\left.\rho_{\text {gold }} \approx 0.95\right)$. That is, the ITO temperature was directly measured from the ITO side while the temperature was also measured through the semi-transparent media. Due to the thinness of the ITO film, the ITO temperatures measured from both sides are expected to be same if the optical properties of the materials used and the governing equation are correct. Since the measured properties of soda-lime glass and sapphire window were verified in Section 4, we only focus on the properties of the ITO. To obtain the boundary condition at the outer surface of the sapphire window, which is required for ITO temperature tracking through the semi-transparent media, a material with high emissivity such as black lacquer or graphite paint (Ted Pella, Inc.) was applied as shown in Figure 7. The measured emissivities of the black lacquer and the graphite paint were 0.976 and 0.75 , respectively.

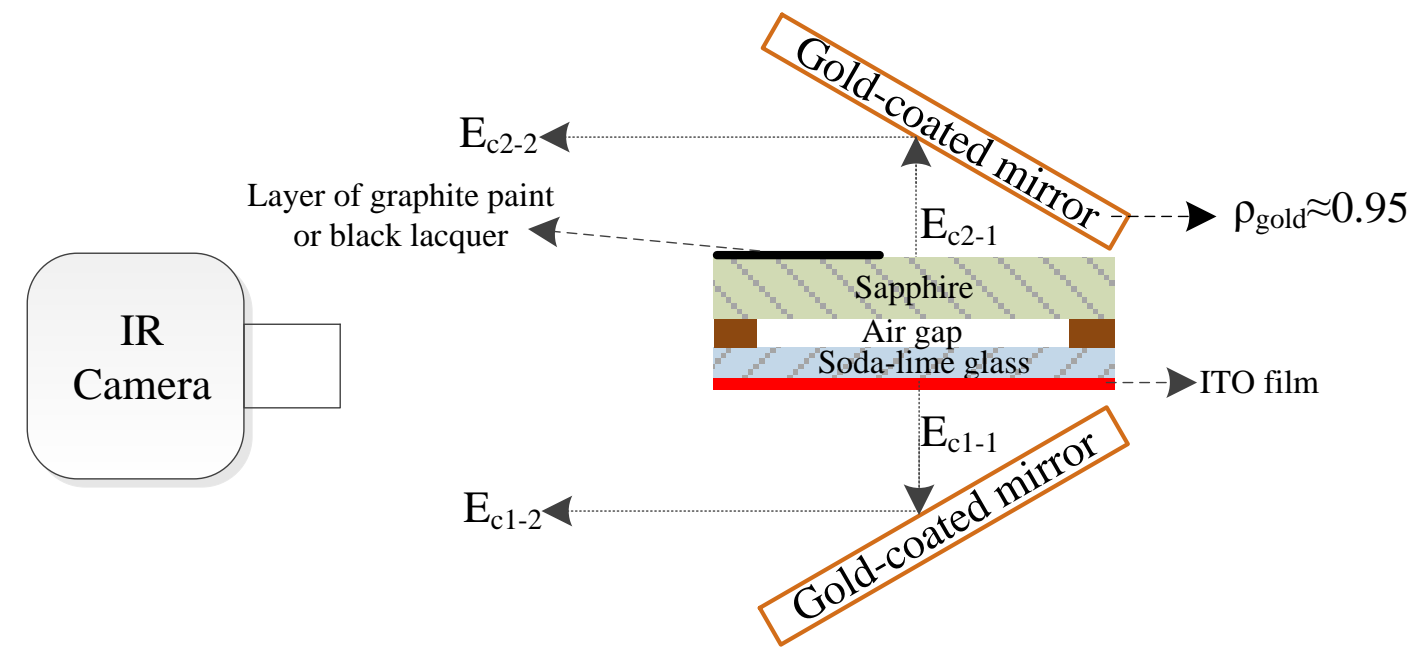

Figure 7. Experimental setup to validate the ITO film's optical properties

The radiation balance equations used to measure the ITO temperature from the ITO side or through the semi-transparent media using the experimental setup shown in Figure 7 can be written as follows:

(i) from the ITO side:

$$
\begin{aligned}
& \mathrm{E}_{\mathrm{cl}-1}=\rho_{\text {Iто }} \mathrm{E}_{\infty}+\left(1-\rho_{\text {IтO }}\right) \mathrm{E}_{\text {гто }} \\
& \mathrm{E}_{\mathrm{cl}-2}=\rho_{\text {gold }} \mathrm{E}_{\mathrm{cl}-1}+\left(1-\rho_{\text {gold }}\right) \mathrm{E}_{\text {gold }}
\end{aligned}
$$


(ii) through the semi-transparent media:

$$
\begin{aligned}
& \mathrm{E}_{\mathrm{c} 2-1}=\rho_{\infty \text {-gold }}^{*} \mathrm{E}_{\infty}+\varepsilon_{\text {sap-gold }}^{*} \mathrm{E}_{\text {sap }}+\varepsilon_{\text {g-gold }}^{*} \mathrm{E}_{\mathrm{g}}+\tau_{\text {ITO-gold }}^{*} \mathrm{E}_{\text {ITO }} \\
& \mathrm{E}_{\mathrm{c} 2-2}=\rho_{\text {gold }} \mathrm{E}_{\mathrm{c} 2-1}+\left(1-\rho_{\text {gold }}\right) \mathrm{E}_{\text {gold }}
\end{aligned}
$$

where $E_{c l-l}$ and $E_{c 2-l}$ represent the total radiation reaching the gold-coated mirrors from the ITO side and through the semi-transparent media, respectively; $E_{c 1-2}$ and $E_{c 2-2}$ are the total radiation measured by the IR camera from each side after being reflected by the gold-coated mirrors (see Figure 7). $\rho_{\text {gold }}$ is the reflectivity of the gold-coated mirror, and $E_{\text {gold }}$ is the energy emitted by the gold-coated mirror $\left(=\sigma F_{3-5 \mu m}(T) T_{\text {gold }}{ }^{4}\right)$. The coefficients used in Eq. (15) are derived in the same way as those in Eq. (1), described in Appendix A.3.

The validation process of the ITO properties is as follows: First, the ITO temperature was observed from both sides for various temperatures of ITO film within a range of $30-85^{\circ} \mathrm{C}$, which was controlled using electrical Joule heating. The measurements at different ITO temperatures were performed under steady-state conditions. By comparing the tracked temperatures from the two sides, the ITO properties (i.e., $\rho_{\text {ITO }}, \varepsilon_{\text {ITO }}=1-\rho_{\text {ITO }}$ ) shown in Table 1 were slightly adjusted so that we could find the optimum properties to ensure the best performance of the present temperature-tracking. As a result, the optimum $\rho_{\text {ITO }}$ was determined as 0.84 , which was a little larger than the nominal value obtained from the optical property measurement (0.82) discussed in Section 4 (see Table 1). Accordingly, the $\varepsilon_{I T O}$ was adjusted based on the relation $\varepsilon_{I T O}=1-\rho_{I T O}$. Then, applying these ITO properties, the ITO temperature measurements under steady-state conditions were performed again, and the temperatures obtained from the two sides were compared, the results of which are shown in Figure 8 (left side). The maximum difference was found to be $1.1{ }^{\circ} \mathrm{C}$, which occurred when the ITO temperature was near $30^{\circ} \mathrm{C}$, the lowest temperature measured in this test. Note that, due to the low emissivity of ITO, the direct temperature measurement from the ITO side may have substantial uncertainty, especially at low ITO temperatures. For instance, small fluctuations in the ambient temperature may considerably affect the temperature tracking results from the ITO side because the surrounding conditions play a dominant role at lower ITO temperatures [see Eq. (13)]. Thus, to reduce measurement uncertainty caused by the ITO properties, a thin graphite paint of higher emissivity $(\varepsilon=0.75)$ was applied to the ITO side. After that, the temperature measurement was performed again as described above. Due to the thinness of the layer of graphite paint, it was expected that the surface temperature of the graphite paint would be almost same as that of the ITO. In Figure 8 (right side), the temperatures measured from both sides are compared. We can see that the largest difference still occurred at the lowest ITO temperature, near $30^{\circ} \mathrm{C}$, but the maximum discrepancy was reduced to 0.6 
${ }^{\circ} \mathrm{C}$. These experimental findings indicate that the ITO properties determined here (i.e., $\rho_{I T O}=0.84, \varepsilon_{I T O}=0.16$ ) are valid enough to be used for the wall temperature tracking in the present study.
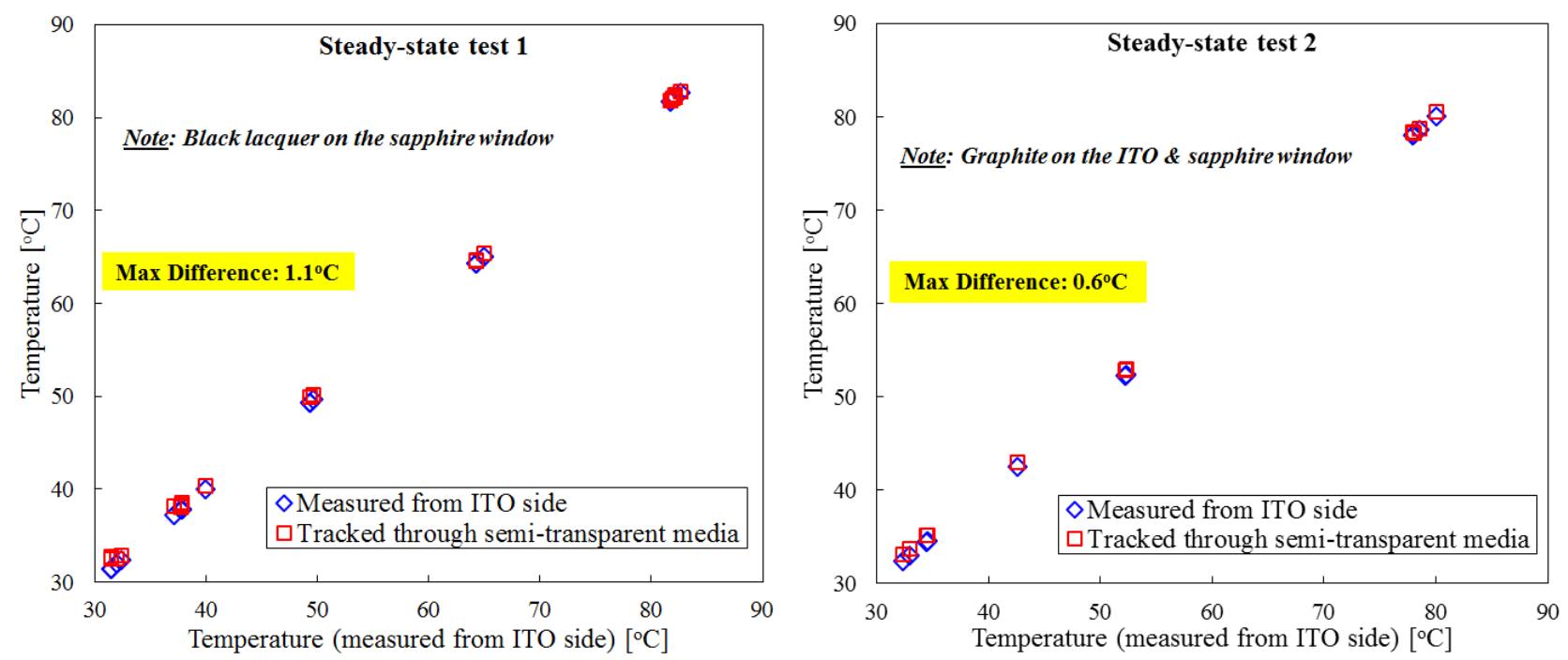

Figure 8. Validation of temperature-tracking algorithm under steady-state conditions

\subsection{Transient test}

The performance of wall temperature tracking algorithm were also tested under transient conditions of the wall (i.e., ITO film) temperature. For the test, virtually the same experimental setup as that used for the steady-state test (Section 5.1) was used, but an air cooling device was added to induce abrupt temperature change on the heater surface as shown in Figure 9. The time-dependent variation of the heater surface temperature was investigated using the IR camera (recording speed: $30 \mathrm{~Hz}$ ) directly from the ITO side as well as through the semi-transparent media, and the temperatures measured from both sides were compared. As discussed in Section 5.1, since the surface temperature directly measured from the ITO side may have considerable uncertainty due to the effect of the ambient temperature, the ITO surface was covered with a thin layer of high-emissive material during the test. 


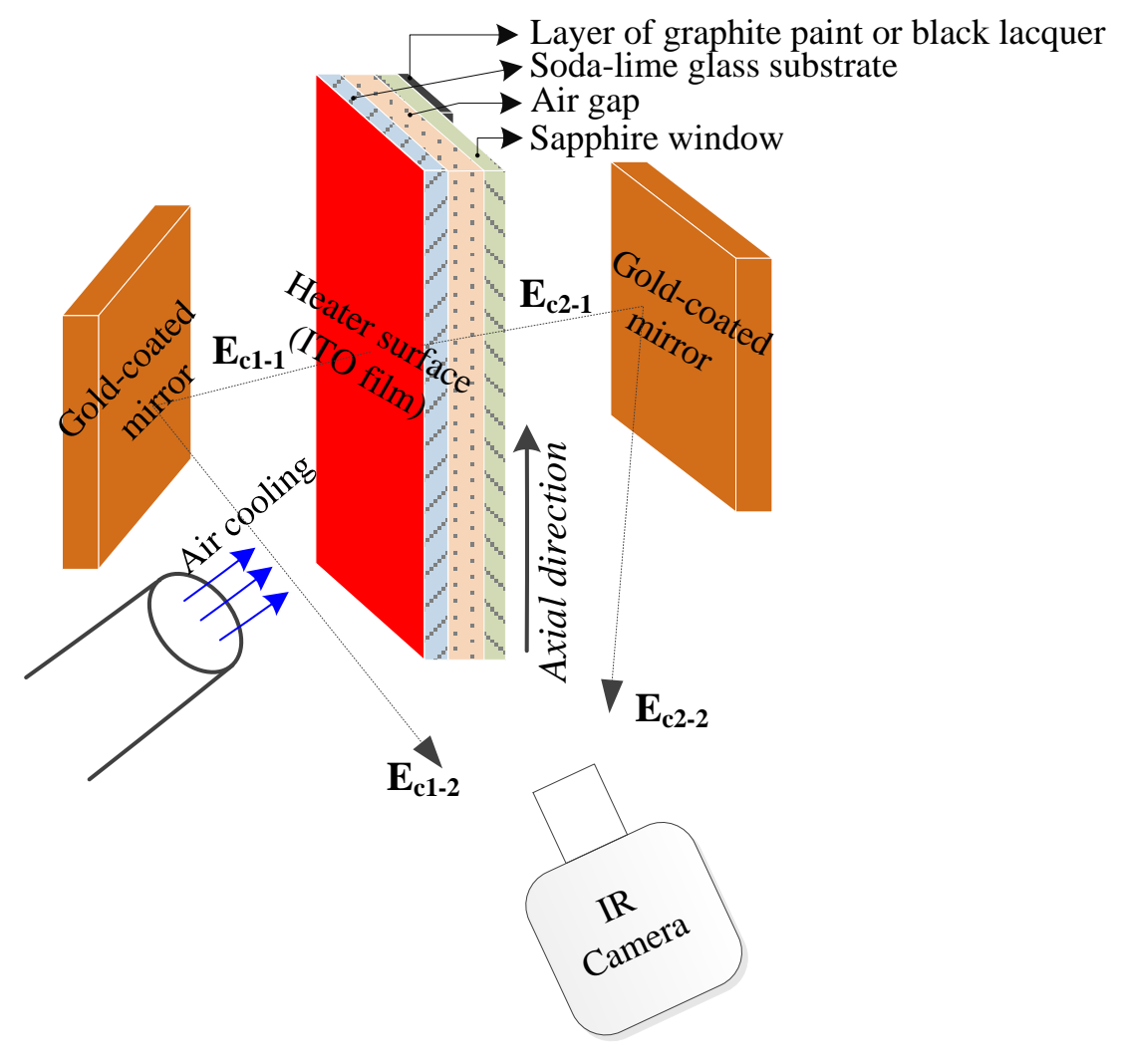

Figure 9. Experimental setup for transient test with cold air injection

The variation of the ITO temperature measured from the two sides was first compared at the local position. Initially, the ITO film was electrically heated, and the surface temperature was kept constant (i.e., steady state) at a certain temperature. Then, cold air was abruptly injected to induce temperature drop on the ITO film. Figure 10 shows the time-dependent variation of the ITO temperature measured from both sides. For the four different tests, which were performed separately, the wall temperatures obtained from the two sides agreed well, implying that temperature tracking through the semi-transparent media was successfully performed. The maximum difference between the direct ITO surface temperature measurement and the temperature measured through the semitransparent media was $0.5^{\circ} \mathrm{C}$ for the all test cases. 

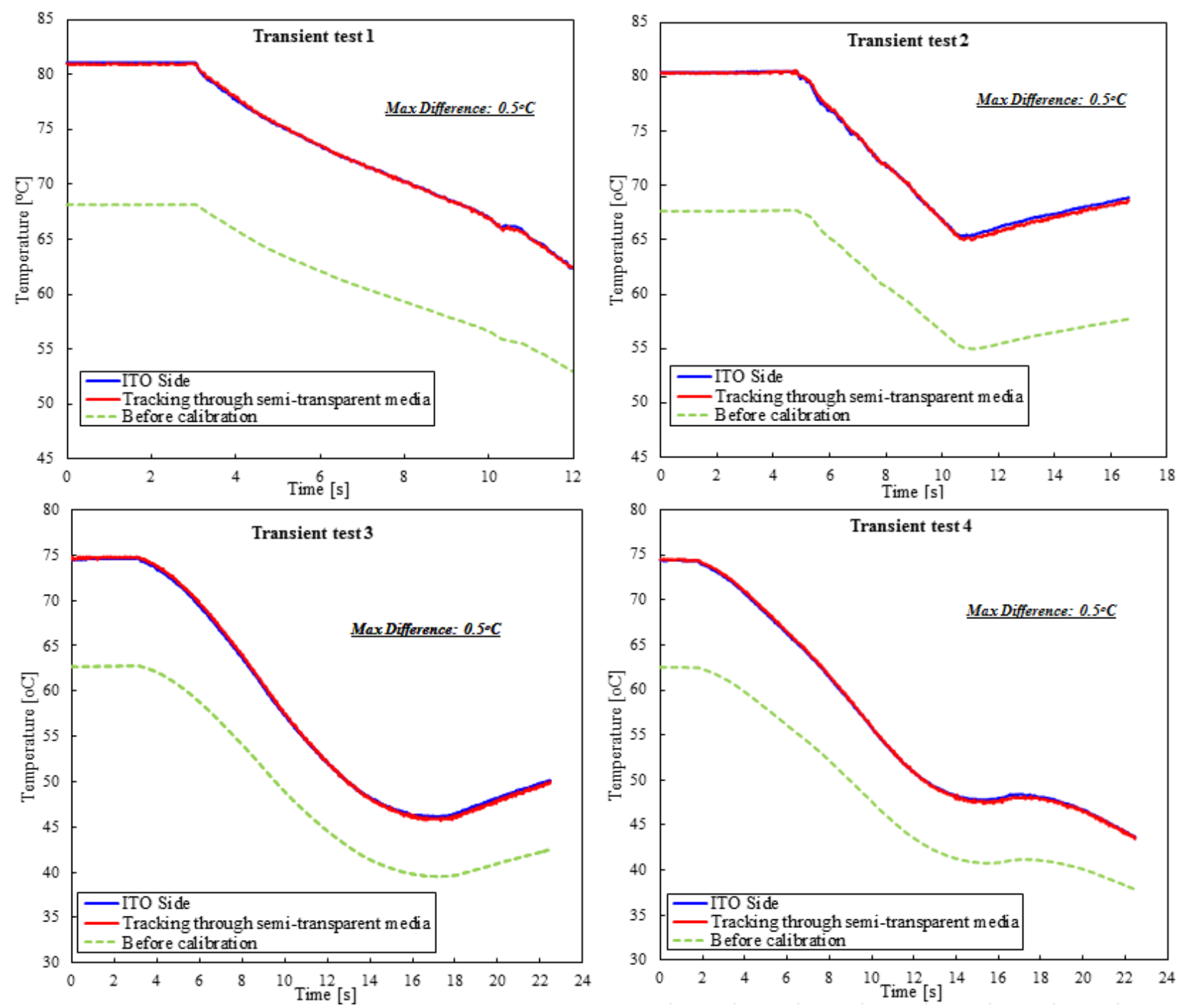

Figure 10. Validation test under transient condition

Note that the above-mentioned wall temperature tracking was performed by coupling the 1D heat conduction equation with the Eq. (1). If the thickness of the glass substrate is thin and the temperature gradient through the glass thickness is much larger than in the other directions, 1D heat conduction can be assumed safely. However, if a significant temperature gradient occurs in other directions due to reasons such as the local boiling phenomenon, the multi-dimensional (multi-D) effect may increase, which can subsequently affect the performance of the present temperature tracking. Therefore, to better understand the multi-D heat conduction effect throughout the media in the present heater wall design, an additional test was performed. Specifically, starting from a steadystate ITO temperature condition, cold air was injected at the bottom of the ITO film to induce a significant 
temperature gradient on the wall in the axial direction. Then, the time-dependent variation of the spatial temperature profile was obtained by the IR camera from the two sides (i.e., directly from the ITO side and through the semitransparent media). In Figure 11, the axial centerline temperature profiles on the ITO film (total axial length $\mathrm{L}_{0}=3$ $\mathrm{cm}$ ) measured from the two sides are compared at several time instances after the injection of cold air. Then, we can see the tracked temperature through the semi-transparent media still agreed well with the temperature directly measured from the ITO side. This means that the multi-D effect of heat conduction was insignificant despite the large temperature gradient in the axial direction and hardly affected the performance of the present wall temperature tracking during this test.

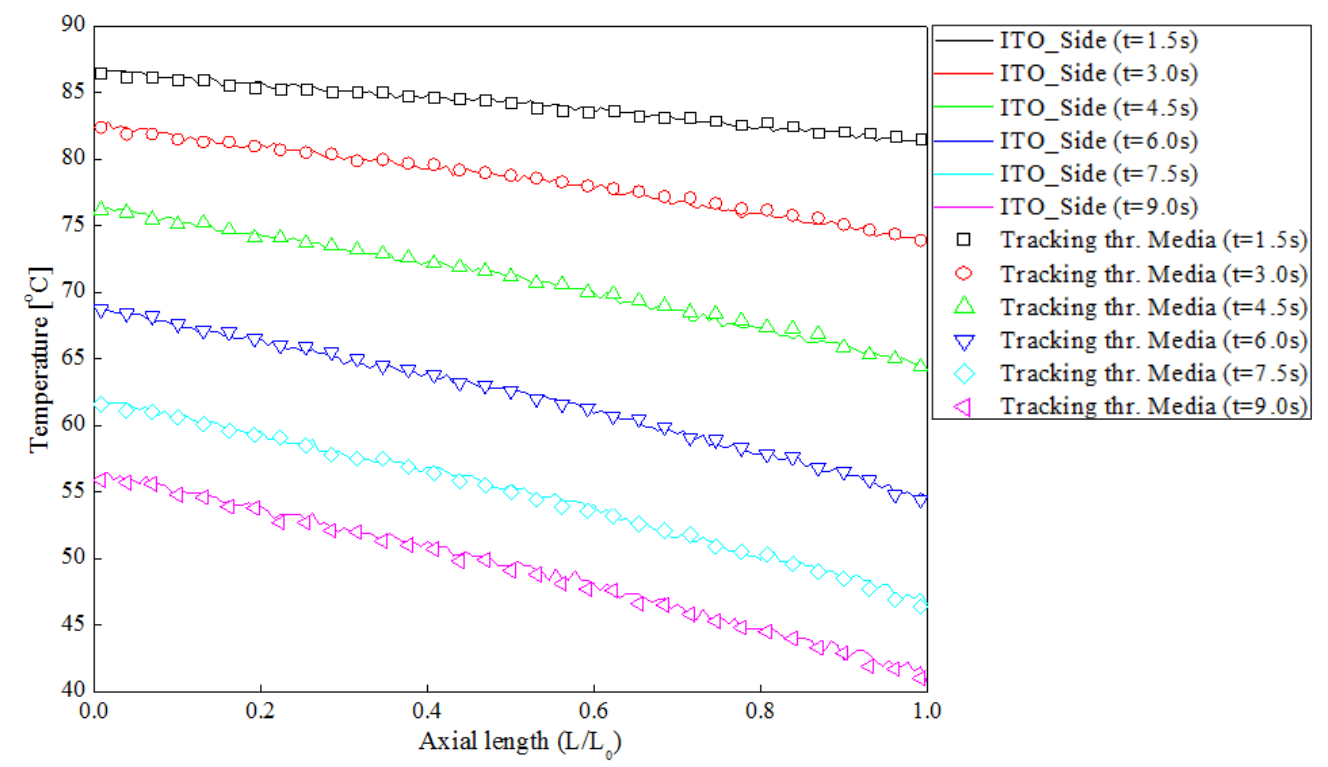

Figure 11. Axial centerline temperature profile variation after the cold air injection $\left(\mathrm{L}_{0}=3 \mathrm{~cm}\right)$

To further confirm the effect of multi-D heat conduction, we compared the axial temperature profiles obtained from the 1D and 2D heat conduction coupling algorithms. In Figure 12, the axial temperature profile at different time instances (i.e., $t=1.52 \mathrm{~s}$ and $\mathrm{t}=9.12 \mathrm{~s}$ ) after the air injection $(\mathrm{t}=0)$ is shown. For the $2 \mathrm{D}$ heat conduction coupling algorithm, heat conduction along the axial direction was additionally considered. On the top and bottom boundaries (i.e., at $\mathrm{L}=0$ and $\mathrm{L}_{0}$ ), the $1 \mathrm{D}$ heat conduction algorithm was used to provide the boundary conditions required for the $2 \mathrm{D}$ heat conduction simulation. Figure 12 shows that the wall temperature profiles 
obtained from the 1D and 2D heat conduction coupling algorithm are almost same, meaning that the multi-D effect of heat conduction did not play a critical role in the wall temperature tracking during this test.
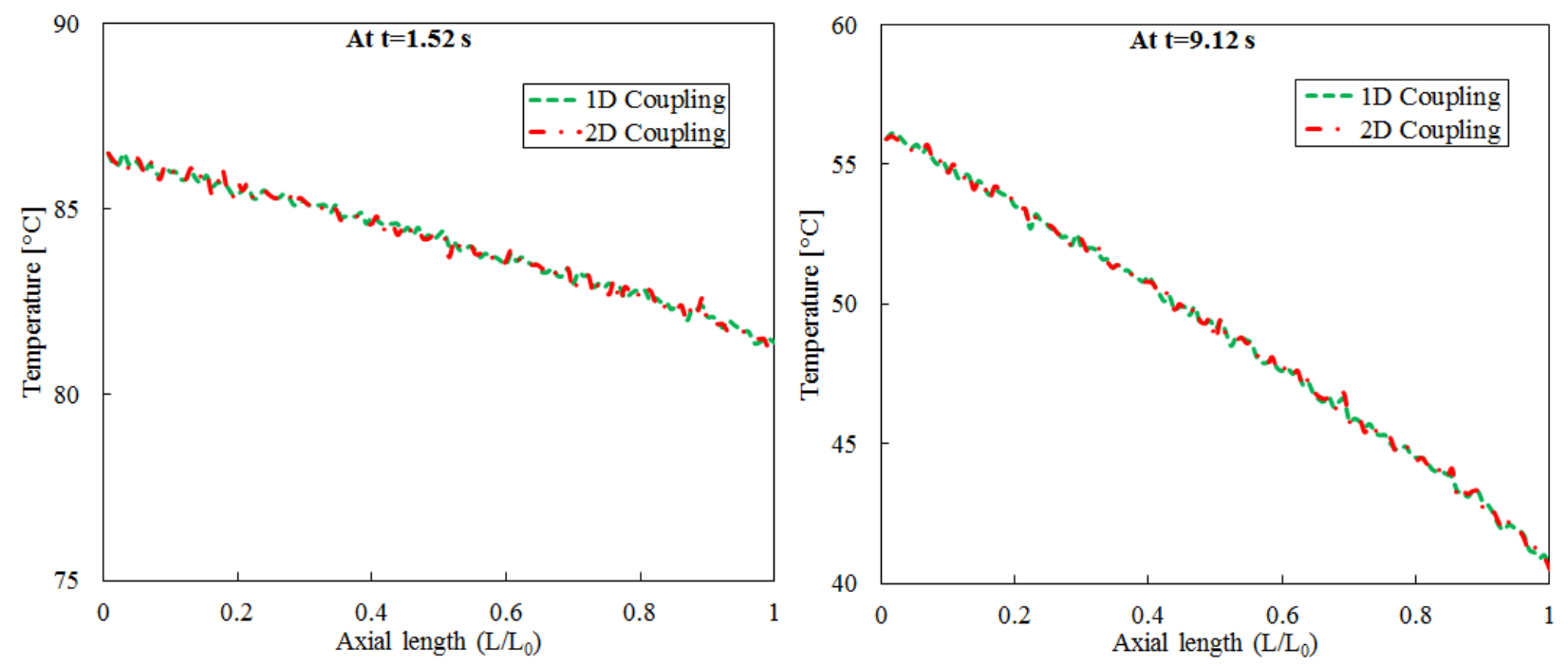

Figure 12. Variation of axial wall temperature profiles based on coupling the $1 \mathrm{D}$ and $2 \mathrm{D}$ heat conduction equations

\subsection{Heat flux measurement}

Subsequent to all the validations for accurate wall temperature tracking described above, we conducted additional tests to confirm the performance of present algorithm for the heat flux measurement as well. Since the present algorithm provides the temperature profile across the multi-layer media as well as the heater surface temperature (see Figure A-6), the amount of heat entering the fluid during the single-phase convection and/or boiling process can also be obtained using this algorithm by considering the heat balance at the wall. In Figure 13, the heat balance relation at the wall (i.e., heater surface) is presented which can be derived by neglecting the temperature gradient across the small thickness of ITO film. This relation implies that the amount of heat entering the fluid $\left(\mathrm{q}_{\text {in }}\right)$ can be determined by estimating the heat conduction through the glass thickness $\left(\mathrm{q}_{\text {cond }}\right)$, the heat retained within the ITO film during the transient process, and the total power provided by the power supply ( $\left.\mathrm{q}_{\mathrm{gen}}\right)$. Obviously, the best way to confirm the validity of such estimation is to compare the results with the separate measurements of $\mathrm{q}_{\mathrm{in}}$. In this sense, we performed the heat loss test using the square test section described in section 2 which was installed in the vertical upward flow boiling test loop (the specific loop configuration is described in section 6). This test was carried out while there was no fluid inside the loop and by keeping track of the heat input 
needed to maintain a given wall temperature at steady state which was monitored by IR camera. Then, the steady state temperatures achieved at various heat inputs were recorded after which the relation between the heat input and the average wall temperature over the heated area was obtained. Assuming that the heat removal due to the natural convection of air was negligible during this test, such relation can be used to estimate the average heat loss through the multi-layer heater wall for the actual flow boiling experiment. Therefore, using this relation, we could obtain the average heat flux entering the fluid $\left(\mathrm{q}_{\text {in }}\right)$ at various flow boiling conditions within the heat flux range $9 \sim 37 \mathrm{~kW} / \mathrm{m}^{2}$ given by power supply (i.e., Measurement 1 in Figure 14). Then, the results are compared with the time-averaged heat fluxes calculated by the present algorithm in which the time-dependent heat storage within the thin ITO film was ignored. This is shown in Figure 14 which indicates that the maximum difference between the two separate measurements for the $\mathrm{q}_{\text {in }}$ was found to be $1.9 \%$. This implies that the present algorithm keeps the validity for the heat flux measurement as well.

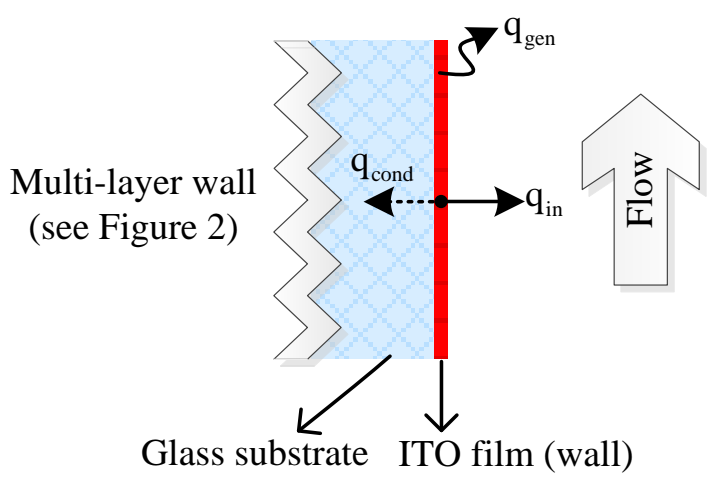

Heat balance relation: $\mathrm{q}_{\mathrm{in}}=\mathrm{q}_{\mathrm{gen}}-\mathrm{q}_{\mathrm{cond}}-\left(\rho \mathrm{c}_{\mathrm{p}}\right)_{\mathrm{w}} \delta_{\mathrm{w}} \frac{\partial \mathrm{T}_{\mathrm{w}}}{\partial \mathrm{t}}$

Figure 13. Heat balance relation at the wall to obtain the local heat input to the fluid $q_{\text {in }}$ 


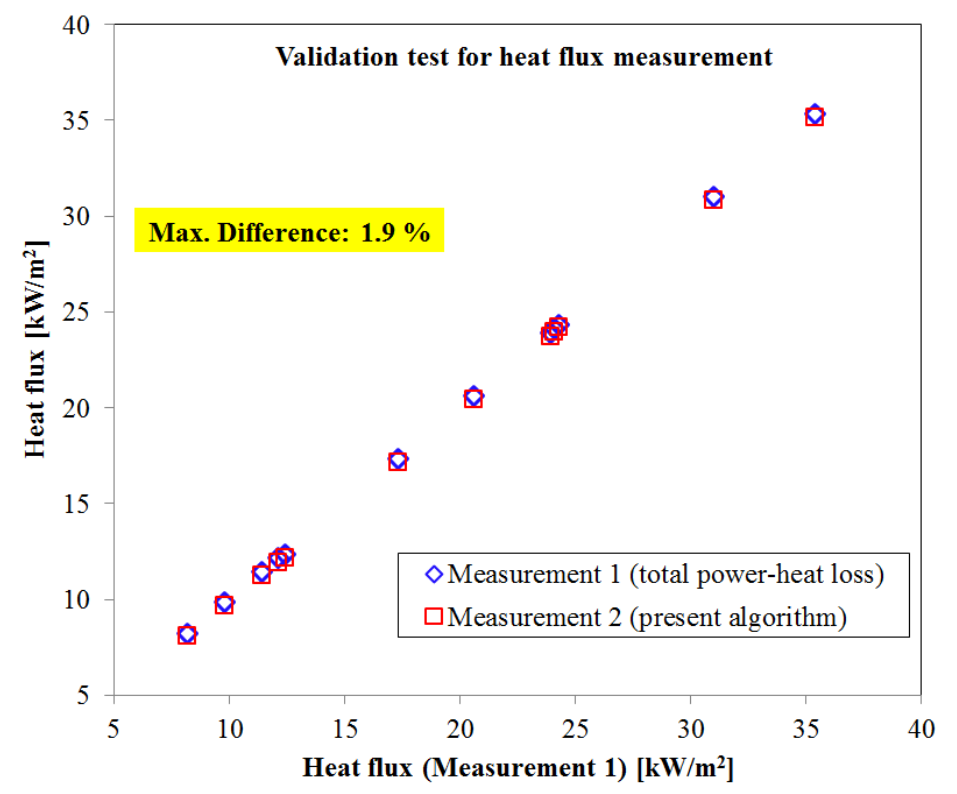

Figure 14. Validation of heat flux measurement using the present algorithm

6. Application in a subcooled flow boiling experiment

Based on the experimental strategy and validations described above, a subcooled flow experiment was performed. The test loop was configured for a working fluid to be pumped upward through the test section of square geometry (see Figure 2) at atmospheric pressure. The temperature of the working fluid to the test section was controlled through a small plate heat exchanger, and the flow rate was adjusted using a control valve placed downstream of a constant speed centrifugal pump. The refrigerant $3 \mathrm{M}^{\mathrm{TM}}$ Novec ${ }^{\mathrm{TM}} 7000$ was used as a working fluid of which boiling point is $34^{\circ} \mathrm{C}$ at atmospheric pressure. Also, an entry length (L) of $\mathrm{L} / \mathrm{D} \approx 61$ was used before the test section inlet so that a hydro-dynamically fully developed flow could be achieved in the measurement section. For this test, only a single nucleation site was created in order to allow a clear view of how such a boiling phenomenon fundamentally affects the heat transfer characteristics on the wall in relation to the vapor bubble motions. In addition, the IR camera was intended to capture the entire heated area to observe the wall heat transfer over the single-phase and two-phase flow regions, as shown in Figure 15. The spatial and temporal resolutions of the thermal images from IR camera were $274 \mu \mathrm{m}$ and $300 \mathrm{~Hz}$, respectively. 


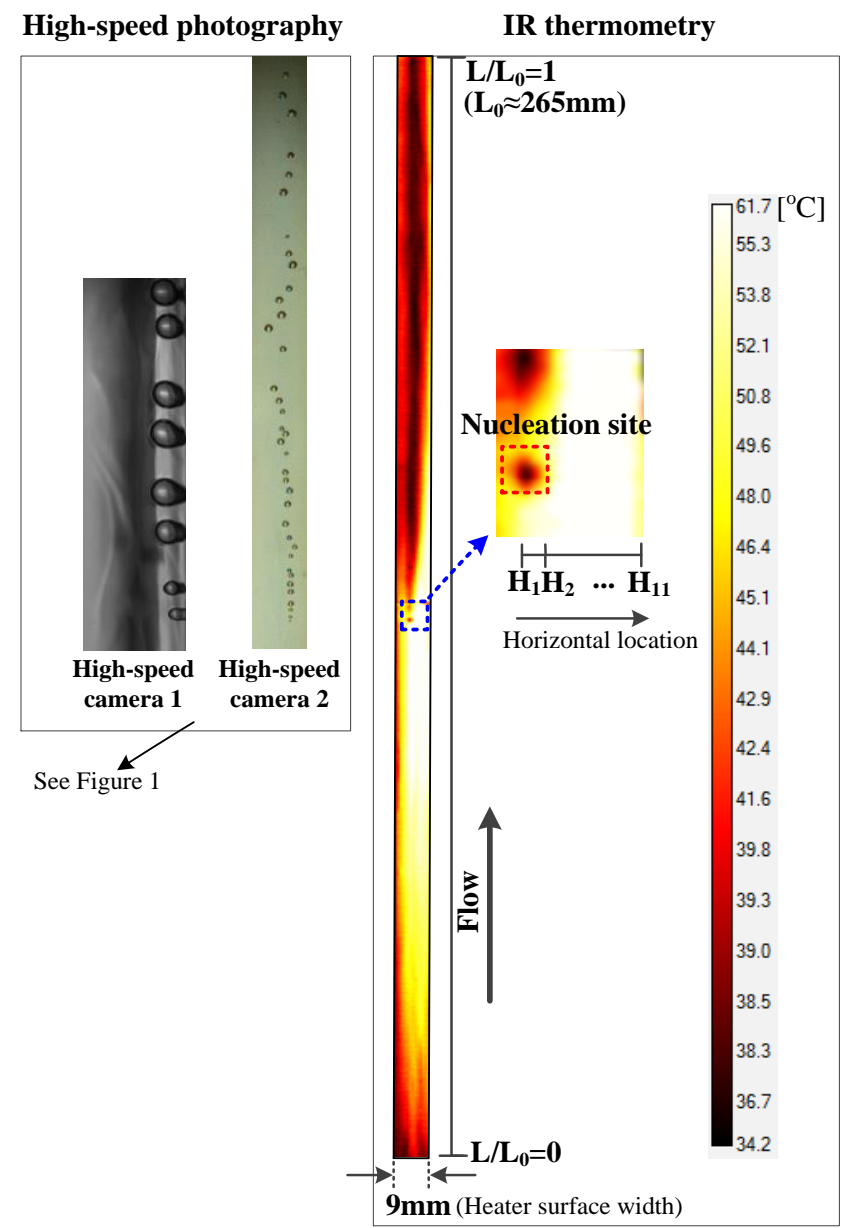

Figure 15. Visual measurement of vapor bubbles and 2D wall temperature map (before calibration) in the vertical upward test section

Representative images captured by high-speed video camera and the 2D wall temperature map from IR thermometry are shown in Figure 15 , which were obtained at a test condition of inlet liquid temperature $=20{ }^{\circ} \mathrm{C}$, heat flux $=13.9 \mathrm{~kW} / \mathrm{m}^{2}$, and liquid mass flux $=216.3 \mathrm{~kg} / \mathrm{m}^{2} \mathrm{~s}$. In Figure 16, the time-averaged centerline wall temperature in the axial direction is shown. We can see that the cooling performance at the heater surface changed drastically due to the appearance of the nucleation site located at $\mathrm{L} / \mathrm{L}_{0} \approx 0.5$. As expected, the wall temperature profile increased almost linearly through the single-phase flow region, but the wall temperature dropped abruptly after the nucleation site because the nucleating and sliding bubbles enhanced the heat removal through evaporation. In particular, at the initial stage of bubble growth, the bubbles grew very fast, inducing a sharp wall temperature drop near the nucleation site. Another important mechanism for this cooling performance is thought to be the 
agitated liquid flow motion induced by the boiling bubbles. However, once the bubble grew up to a certain size at which the heat transfer between the liquid and vapor phases became more balanced $\left(\mathrm{L} / \mathrm{L}_{0} \geq 0.6\right)$, the wall temperature gradient in axial direction significantly reduced. Figure 17 shows the horizontal temperature profile of the wall (from $\mathrm{H}_{1}$ to $\mathrm{H}_{11}$ ) at different axial locations (from $\mathrm{L} / \mathrm{L}_{0}=0.1$ to $\mathrm{L} / \mathrm{L}_{0}=1$ ). The nucleation site appeared near the left edge of the heater surface $\left(\mathrm{H}_{1}\right)$ just below the middle of the test section in the axial direction $\left(\mathrm{L} / \mathrm{L}_{0} \approx 0.5\right.$, see Figure 13). In Figure 17, the horizontal temperature profiles at the axial locations upstream and downstream of the nucleation site is drawn with the 'non-filled' and 'filled' symbols, respectively. The abrupt temperature drop on the wall where the nucleation site appeared (i.e., $\mathrm{H}_{1}$ at $\mathrm{L} / \mathrm{L}_{0}=0.5$ ) is clear and the nucleation caused the large temperature gradient in the horizontal direction at that axial location. Interestingly, this temperature gradient in the horizontal direction diminished rapidly downstream of the nucleation site (i.e., $L / \mathrm{L}_{0} \geq 0.6$ ). This can be explained by the observation of vapor bubble motions obtained from the high-speed video cameras. In particular, viewed from the top of the vapor bubbles (i.e., from high-speed camera 2 in Figure 15), we could clearly see that the horizontal waving motions of the bubbles were enhanced as the bubbles slid upward; whereas, such waving motions were not found near the nucleation site. Consequently, the heat removal due to the sliding bubbles occurred over a wider region at upper locations due to the bubbles' wider trajectory. For this reason, the wall temperature profile became flatter downstream of the nucleation site in Figure 17. 


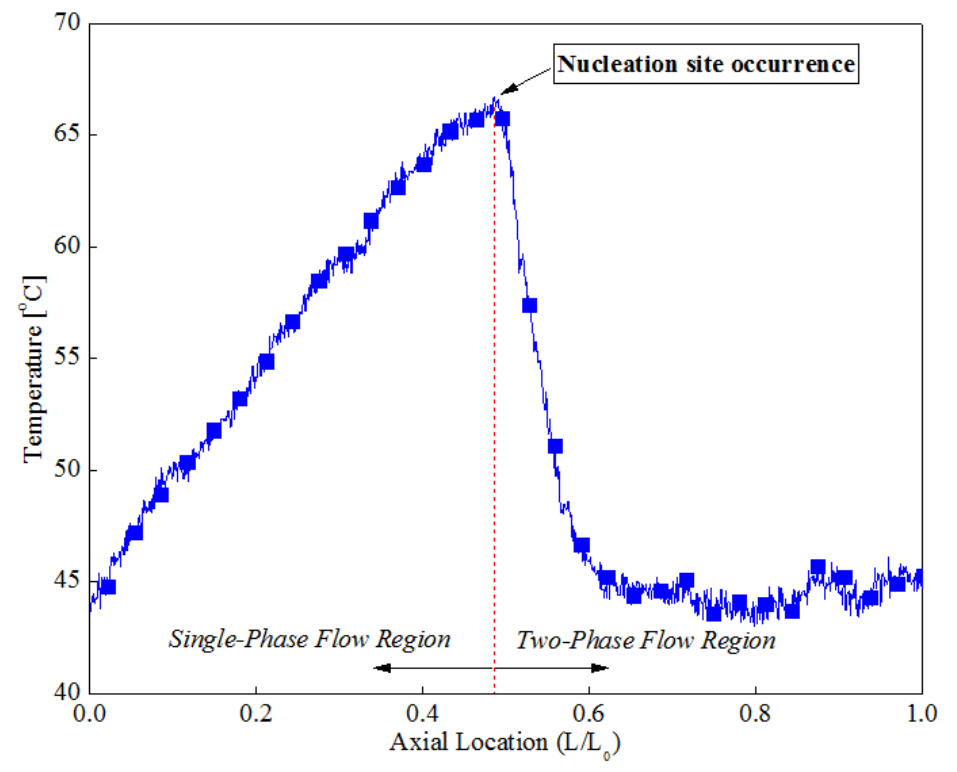

Figure 16. Axial distribution of wall temperature at the centerline

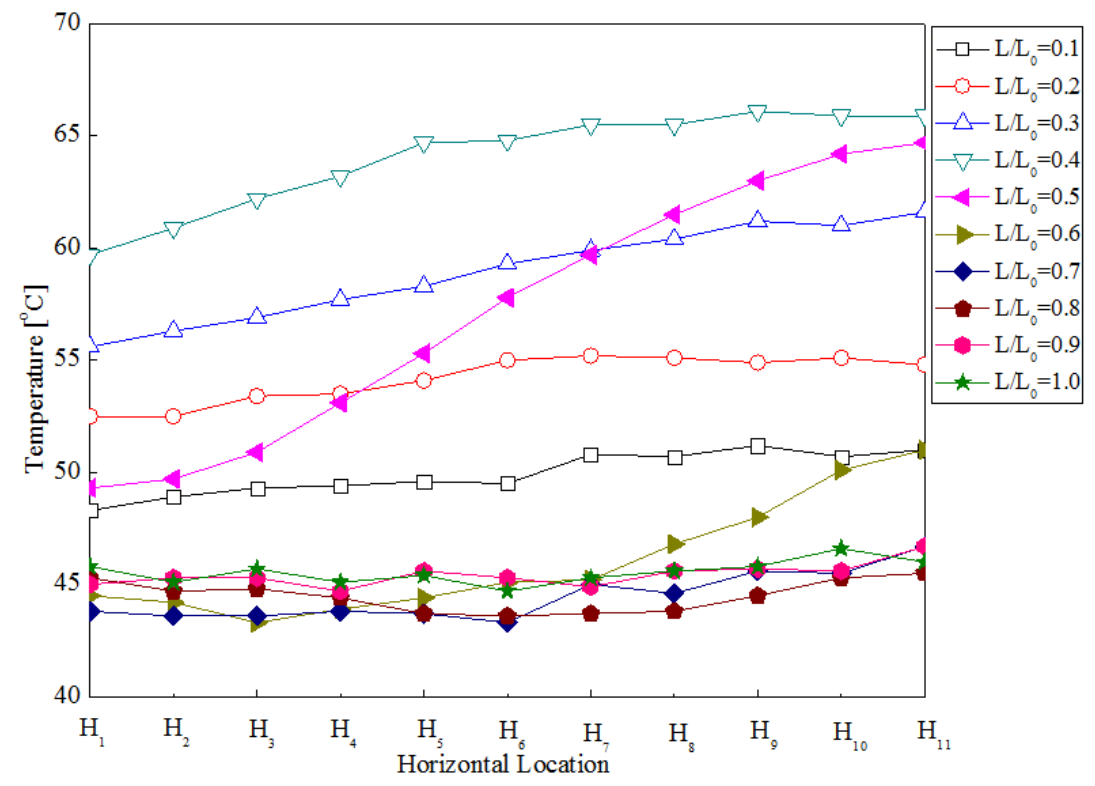

Figure 17. Horizontal wall temperature profiles at different axial locations (non-filled symbols: axial locations upstream of the nucleation site; filled symbols: axial locations downstream of the nucleation site)

\section{Uncertainty quantification}

The uncertainty of the present temperature tracking depends on the uncertainties associated with the uncertainty of individual parameters used for the tracking, e.g., optical properties, thermal properties, and ambient 
temperature. The major sources of uncertainty considered are listed in Table 2. These parameters were determined by investigating each parameter's sensitivity in the present temperature tracking method. That is, only parameters of primary significance were selected. The ITO film's optical properties were not considered here because they were obtained in a deterministic way through the validation process (see Section 5.1). We found that the amount of uncertainty due to the ambient temperature $\left(\approx 0.34^{\circ} \mathrm{C}\right)$ hardly affected the temperature tracking result when the wall temperature tracking is performed using the proposed heater wall design. Thus, the ambient effect is not included in Table 2. However, it is noted that this should not be the case for the direct temperature measurement of ITO surface due to the low emissivity of ITO. For the uncertainty of air's thermal conductivity, possible variations in the air temperature between $20-80^{\circ} \mathrm{C}$ were considered.

Table 2. Major sources of uncertainty for the wall temperature tracking

\begin{tabular}{|c|c|c|}
\hline Parameter & Nominal Value & Uncertainty \\
\hline Apparent transmissivity of glass $\left(\tau_{a p p, g-\infty}\right)$ & & \multirow{4}{*}{ See Table 1.} \\
\hline Apparent reflectivity of glass $\left(\rho_{a p p, g-\infty}\right)$ & & \\
\hline Apparent transmissivity of sapphire $\left(\tau_{a p p, s a p-\infty}\right)$ & & \\
\hline Apparent reflectivity of sapphire $\left(\rho_{a p p, s a p-\infty}\right)$ & & \\
\hline Thermal conductivity of glass & $0.94(\mathrm{~W} / \mathrm{m}-\mathrm{K})$ & $0.01(\mathrm{~W} / \mathrm{m}-\mathrm{K})$ \\
\hline Thermal conductivity of air & $0.026(\mathrm{~W} / \mathrm{m}-\mathrm{K})$ & $0.004(\mathrm{~W} / \mathrm{m}-\mathrm{K})$ \\
\hline
\end{tabular}

After determining the uncertainty parameters, their uncertainty values, and the sensitivity in the present temperature tracking method, the overall uncertainty was calculated by combining the individual parameters' uncertainties using the following equation (Kline and McClintock, 1953):

$$
\mathrm{U}_{\mathrm{T}}=\sqrt{\sum_{\mathrm{i}=1}^{\mathrm{M}}\left(\frac{\partial \mathrm{T}}{\partial \mathrm{V}_{\mathrm{i}}} \mathrm{U}_{\mathrm{V}_{\mathrm{i}}}\right)^{2}}
$$

where $U_{T}$ is the uncertainty of the present temperature measurement, $V_{i}$ is the $\mathrm{i}^{\text {th }}$ uncertainty parameter considered, $M$ is the total number of uncertainty parameters considered, and $\partial T / \partial V_{i}$ and $U_{V i}$ are the sensitivity coefficient and uncertainty for $V_{i}$, respectively. The uncertainty associated with the heater surface temperature measurement using the present heater wall design was then estimated as $0.7^{\circ} \mathrm{C}$. Using the similar way, the uncertainty of heat flux measurement was estimated as $0.6 \mathrm{~kW} / \mathrm{m}^{2}$. 


\section{Conclusions}

An experimental strategy to achieve both enhanced flow visualization and accurate wall temperature/heat flux measurement in a convective boiling system has been described. High-speed photography and IR thermometry were employed as experimental techniques, and the feasibility of applying both techniques to a forced convective boiling experiment which involves a large heated area was successfully demonstrated with extensive validations.

We expect that the present approach will help to achieve the more useful insight into the boiling heat transfer mechanism, e.g., the effects of vapor bubbles on the heat transfer as they develop throughout the heated flow channel. In particular, the enhanced reliability of measurements using optical techniques would help to uncover the detailed relation between the bubble dynamics and the wall heat transfer. Also, it is expected that the flow visualization techniques such as particle image velocimetry (PIV) can be applied in the current experimental facility, which will allow the additional information about the liquid phase.

\section{Acknowledgment}

The authors acknowledge the financial support provided by the Consortium for Advanced Simulation of Light Water Reactors (CASL) for this research. 


\section{References}

Barber, J., Sefiane, K., Brutin, D., Tadrist, L., 2009. Hydrodynamics and heat transfer during flow boiling instabilities in a single microchannel. Appl Therm Eng 29, 1299-1308.

Bayazit, B.B., Hollingsworth, D.K., Witte, L.C., 2003. Heat transfer enhancement caused by sliding bubbles. J Heat Trans-T Asme 125, 503-509.

Boye, H., Staate, Y., Schmidt, J., 2007. Experimental investigation and modelling of heat transfer during convective boiling in a minichannel. Int J Heat Mass Tran 50, 208-215.

Chu, I.C., No, H.C., Song, C.H., 2011. Bubble Lift-off Diameter and Nucleation Frequency in Vertical Subcooled Boiling Flow. J Nucl Sci Technol 48, 936-949.

Diaz, M.C., Boye, H., Hapke, I., Schmidt, J., Staate, Y., Zhekov, Z., 2006. Investigation of flow boiling in narrow channels by thermographic measurement of local wall temperatures. Microfluid Nanofluid 2, 1-11.

Estrada-Perez, C.E., Hassan, Y.A., 2010. PTV experiments of subcooled boiling flow through a vertical rectangular channel. Int J Multiphas Flow 36, 691-706.

Fischer, S., Herbert, S., Sielaff, A., Slomski, E.M., Stephan, P., Oechsner, M., 2012. Experimental Investigation of Nucleate Boiling on a Thermal Capacitive Heater Under Variable Gravity Conditions. Microgravity Sci Tec 24, 139-146.

Gerardi, C., Buongiorno, J., Hu, L.W., McKrell, T., 2010. Study of bubble growth in water pool boiling through synchronized, infrared thermometry and high-speed video. Int J Heat Mass Tran 53, 4185-4192.

Golobic, I., Petkovsek, J., Baselj, M., Papez, A., Kenning, D., 2009. Experimental determination of transient wall temperature distributions close to growing vapor bubbles. Heat Mass Transfer 45, 857-866.

Hapke, I., Boye, H., Schmidt, J., 2000. Onset of nucleate boiling in minichannels. Int J Therm Sci 39, $505-513$.

Horny, N., 2003. FPA camera standardisation. Infrared Phys Techn 44, 109-119.

Incropera, F.P., Dewitt, D.P., 2002. Introduction to heat transfer. John Wiley \& Sons, Inc.

Jung, J., Kim, S.J., Kim, J., 2014. Observations of the Critical Heat Flux Process During Pool Boiling of FC-72. J

Heat Trans-T Asme 136

Kenning, D.B.R., Kono, T., Wienecke, M., 2001. Investigation of boiling heat transfer by liquid crystal thermography. Exp Therm Fluid Sci 25, 219-229.

Kim, T.H., Kommer, E., Dessiatoun, S., Kim, J., 2012. Measurement of two-phase flow and heat transfer parameters using infrared thermometry. Int J Multiphas Flow 40, 56-67.

Klausner, J.F., Mei, R., Bernhard, D.M., Zeng, L.Z., 1993. Vapor Bubble Departure in Forced-Convection Boiling. Int J Heat Mass Tran 36, 651-662.

Kline, S.J., McClintock, F.A., 1953. Describing Uncertainties in Single-Sample Experiments. Mechanical Engineering 75, 3-8.

Kunkelmann, C., Ibrahem, K., Schweizer, N., Herbert, S., Stephan, P., Gambaryan-Roisman, T., 2012. The effect of three-phase contact line speed on local evaporative heat transfer: Experimental and numerical investigations. Int $\mathrm{J}$ Heat Mass Tran 55, 1896-1904. 
Muwanga, R., Hassan, I., 2006. Local heat transfer measurements in microchannels using liquid crystal thermography: Methodology development and validation. J Heat Trans-T Asme 128, 617-626.

Okawa, T., Ishida, T., Kataoka, I., Mori, M., 2005. Bubble rise characteristics after the departure from a nucleation site in vertical upflow boiling of subcooled water. Nucl Eng Des 235, 1149-1161.

Schweizer, N., Stephan, P., 2009. Experimental Study of Bubble Behavior and Local Heat Flux in Pool Boiling Under Variable Gravitational Conditions. Multiphase Science and Technology 21, 329-350.

Situ, R., Ishii, M., Hibiki, T., Tu, J.Y., Yeoh, G.H., Mori, M., 2008. Bubble departure frequency in forced convective subcooled boiling flow. Int J Heat Mass Tran 51, 6268-6282.

Theofanous, T.G., Dinh, T.N., Tu, J.P., Dinh, A.T., 2002a. The boiling crisis phenomenon - Part II: dryout dynamics and burnout. Exp Therm Fluid Sci 26, 793-810.

Theofanous, T.G., Tu, J.P., Dinh, A.T., Dinh, T.N., 2002b. The boiling crisis phenomenon - Part I: nucleation and nucleate boiling heat transfer. Exp Therm Fluid Sci 26, 775-792.

Trujillo, M.F., Alvarado, J., Gehring, E., Soriano, G.S., 2011. Numerical Simulations and Experimental Characterization of Heat Transfer From a Periodic Impingement of Droplets. J Heat Trans-T Asme 133.

Vollmer, M., Mollmann, K.-P., 2011. Infrared Thermal Imaging: Fundamentals, Research and APplications. John Wiley \& Sons. 


\section{Appendices}

A.1. Derivation of apparent reflectivity and apparent transmissivity

In Figure A-1, the semi-transparent medium ' $m$ ' is located in the middle of the medium ' $\infty$ '. Consider that the incoming radiation $E_{i n c}$ hits the left side of ' $m$ - $\infty$ ' interface. Then, part of $E_{i n c}$ is reflected at this interface; the reflected amount is $\rho_{m-\infty} E_{\text {inc }}$ while the remainder $\left(1-\rho_{m-\infty}\right) E_{i n c}$ passes through the interface and subsequently reaches the other side of the ' $m-\infty$ ' interface. The energy reaching this ' $m-\infty$ ' interface on the right side is attenuated by the transmissivity of the medium $\left(1-\rho_{m-\infty}\right) \tau_{m} E_{\text {inc }}$. Part of this energy is reflected again at this interface (' $m-\infty$ '), the amount of which is $\rho_{m-\infty}\left(1-\rho_{m-\infty}\right) \tau_{m} E_{i n c}$ while the remainder $\left(1-\rho_{m-\infty}\right)^{2} \tau_{m} E_{i n c}$ passes through this interface. The total amount of reflection and transmission by the semi-transparent medium can be estimated by considering these infinite number of reflections and transmissions within the medium, from which apparent reflectivity $\left(\rho_{a p p, m-\infty}\right)$ and apparent transmissivity $\left(\tau_{a p p, m-\infty}\right)$ can be obtained. As illustrated in Figure A-1, $\rho_{a p p, m-\infty}$ and $\tau_{a p p, m-\infty}$ can be calculated using the analytical form of the infinite series as follows:

- Total energy reflected by the semi-transparent medium ' $m$ '

$$
\begin{aligned}
& {\left[\rho_{\mathrm{m}-\infty}+\left(1-\rho_{\mathrm{m}-\infty}\right)^{2} \rho_{\mathrm{m}-\infty} \tau_{\mathrm{m}}^{2}+\left(1-\rho_{\mathrm{m}-\infty}\right)^{2} \rho_{\mathrm{m}-\infty}^{3} \tau_{\mathrm{m}}^{4}+\left(1-\rho_{\mathrm{m}-\infty}\right)^{2} \rho_{\mathrm{m}-\infty}^{5} \tau_{\mathrm{m}}^{6} \cdots\right] \mathrm{E}_{\mathrm{inc}}} \\
& =\left[\rho_{\mathrm{m}-\infty}+\frac{\left(1-\rho_{\mathrm{m}-\infty}\right)^{2} \rho_{\mathrm{m}-\infty} \tau_{\mathrm{m}}^{2}}{1-\rho_{\mathrm{m}-\infty}^{2} \tau_{\mathrm{m}}^{2}}\right] \mathrm{E}_{\mathrm{inc}} \\
& =\rho_{\text {app } \mathrm{m}-\infty} \mathrm{E}_{\text {inc }}
\end{aligned}
$$

- Total energy transmitted through the semi-transparent medium ' $m$ '

$$
\begin{aligned}
& {\left[\left(1-\rho_{\mathrm{m}-\infty}\right)^{2} \tau_{\mathrm{m}}+\left(1-\rho_{\mathrm{m}-\infty}\right)^{2} \rho_{\mathrm{m}-\infty}^{2} \tau_{\mathrm{m}}^{3}+\left(1-\rho_{\mathrm{m}-\infty}\right)^{2} \rho_{\mathrm{m}-\infty}^{4} \tau_{\mathrm{m}}^{5}+\cdots\right] \mathrm{E}_{\mathrm{inc}}} \\
& =\left[\frac{\left(1-\rho_{\mathrm{m}-\infty}\right)^{2} \tau_{\mathrm{m}}}{1-\rho_{\mathrm{m}-\infty}^{2} \tau_{\mathrm{m}}^{2}}\right] \mathrm{E}_{\mathrm{inc}} \\
& =\tau_{\mathrm{app}, \mathrm{m}-\infty} \mathrm{E}_{\mathrm{inc}}
\end{aligned}
$$




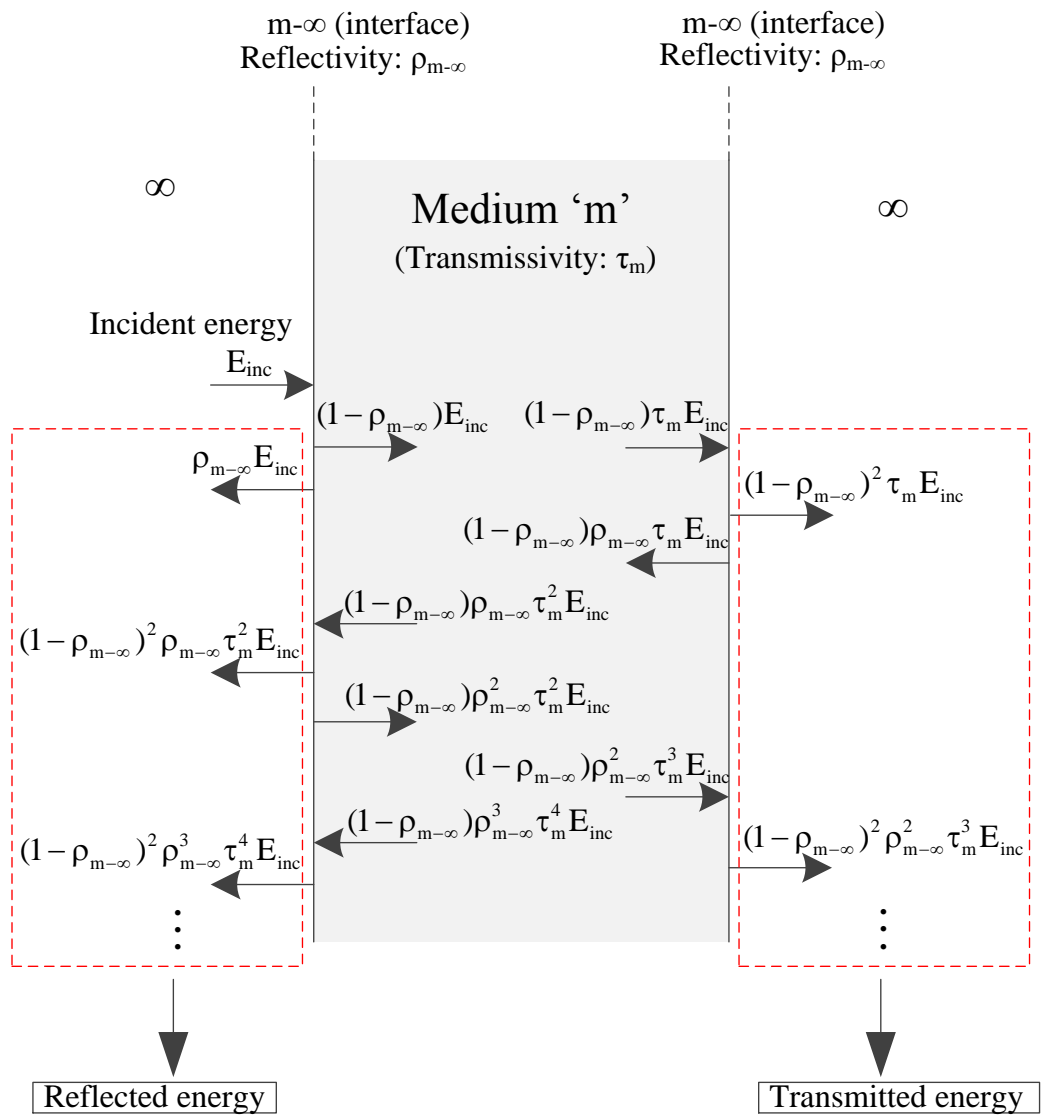

Figure A-1. Energy reflection and transmission throughout the semi-transparent medium 
A.2. Radiation balance equation and coefficients required for validation tests in Section 4.2

Figure A-2 shows the experimental setup used in the validation test with a single layer of semi-transparent medium. The blackbody source temperature is observed through the semi-transparent medium ' $m$ ' (i.e., soda-lime glass, sapphire) using the IR camera. Note that the apparent reflectivity at interface $1, \rho_{a p p, m-\infty, 1}$ as shown in Figure A-2 is considered to be the same as normal reflectivity $\rho_{m-\infty}$ because the reflection from the blackbody source is negligible due to the special coating applied to its surface.

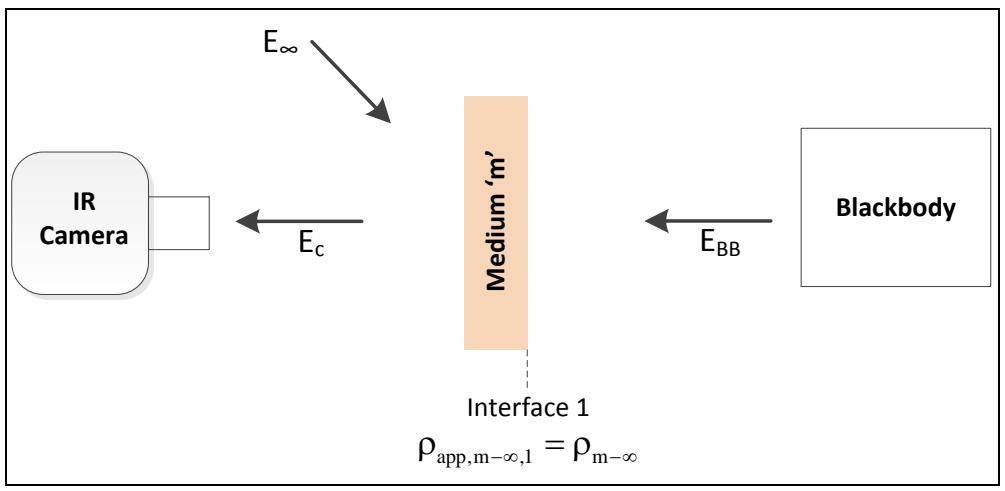

Figure A-2. Experimental setup for validation test with a single layer of medium

The governing equation as well as the derivation of coefficients used to track the blackbody source temperature through the medium is described in Figure A-3. In it, $E_{c}$ represents the total amount of energy reaching the IR camera. The first term on the right-hand side of the balance equation is the energy due to the background radiation reflected by the target objects. The second term is the energy due to the emission from the medium, and the third term is the energy contribution from the blackbody source through the medium. In Figures A-4 and A-5, the experimental set up, governing equation, and the coefficients for the double-layer validation test are also shown. The governing equation and coefficients are obtained using the same principle and assumption mentioned in the singlelayer validation test. The apparent reflectivities at different interfaces are obtained as shown in Figure A-4, which are required to simplify the derivation process of the coefficients shown in Figure A-5. 
Energy Balance Equation: $\mathrm{E}_{\mathrm{c}}=\rho_{\mathrm{app}, \mathrm{m}-\infty}^{*} \mathrm{E}_{\infty}+\varepsilon_{\mathrm{app}, \mathrm{m}-\infty}^{*} \mathrm{E}_{\mathrm{m}}+\tau_{\mathrm{app}, \mathrm{m}-\infty}^{*} \mathrm{E}_{\mathrm{BB}}$ where $\mathrm{E}_{\mathrm{BB}}=\varepsilon_{\mathrm{L}_{\mathrm{m}}} \sigma \mathrm{F}_{3-5 \mu \mathrm{m}}(\mathrm{T}) \mathrm{T}_{\mathrm{BB}}^{4}, \mathrm{E}_{\infty}=\sigma \mathrm{F}_{3-5 \mu \mathrm{m}}(\mathrm{T}) \mathrm{T}_{\infty}^{4}$ $\begin{aligned} \rho_{\mathrm{app}, \mathrm{m}-\infty}= & \rho_{\mathrm{m}-\infty}+\left(1-\rho_{\mathrm{m}-\infty}\right)^{2} \tau_{\mathrm{m}}^{2} \rho_{\mathrm{app}, \mathrm{m}-\infty, 1}+\left(1-\rho_{\mathrm{m}-\infty}\right)^{2} \rho_{\mathrm{m}-\infty} \tau_{\mathrm{m}}^{4} \rho_{\mathrm{app}, \mathrm{m}-\infty, 1}^{2}+\ldots \\ = & \rho_{\mathrm{m}-\infty}+\frac{\left(1-\rho_{\mathrm{m}-\infty}\right)^{2} \tau_{\mathrm{m}}^{2} \rho_{\mathrm{app}, \mathrm{m}-\infty, 1}}{1-\rho_{\mathrm{m}-\infty} \rho_{\mathrm{app}, \mathrm{m}-\infty, 1} \tau_{\mathrm{m}}^{2}} \\ \varepsilon_{\mathrm{app}, \mathrm{m}-\infty}^{*}= & {\left[\rho_{\mathrm{app}, \mathrm{m}-\infty, 1} \tau_{\mathrm{m}}\left(1-\rho_{\mathrm{m}-\infty}\right)+\rho_{\mathrm{app}, \mathrm{m}-\infty, 1}^{2} \tau_{\mathrm{m}}^{3} \rho_{\mathrm{m}-\infty}\left(1-\rho_{\mathrm{m}-\infty}\right)+\ldots\right] } \\ & +\left[\left(1-\rho_{\mathrm{m}-\infty}\right)+\rho_{\mathrm{m}-\infty} \rho_{\mathrm{app}, \mathrm{m}-\infty, 1} \tau_{\mathrm{m}}^{2}\left(1-\rho_{\mathrm{m}-\infty}\right)+\rho_{\mathrm{m}-\infty}^{2} \rho_{\mathrm{app}, \mathrm{m}-\infty, 1}^{2} \tau_{\mathrm{m}}^{4}\left(1-\rho_{\mathrm{m}-\infty}\right)+\ldots\right] \\ = & \frac{\rho_{\mathrm{app}, \mathrm{m}-\infty, 1} \tau_{\mathrm{m}}\left(1-\rho_{\mathrm{m}-\infty}\right)}{1-\rho_{\mathrm{app}, \mathrm{m}-\infty, 1} \rho_{\mathrm{m}-\infty} \tau_{\mathrm{m}}^{2}}+\frac{\left(1-\rho_{\mathrm{m}-\infty}\right)}{1-\rho_{\mathrm{app}, \mathrm{m}-\infty, 1} \rho_{\mathrm{m}-\infty} \tau_{\mathrm{m}}^{2}} \\ = & \frac{\left(1-\rho_{\mathrm{m}-\infty}\right)\left(1+\rho_{\mathrm{app}, \mathrm{m}-\infty, 1} \tau_{\mathrm{m}}\right)}{1-\rho_{\mathrm{m}-\infty} \rho_{\mathrm{app}, \mathrm{m}-\infty, 1} \tau_{\mathrm{m}}^{2}} \\ \tau_{\mathrm{app}, \mathrm{m}-\infty}^{*}= & \left(1-\rho_{\mathrm{m}-\infty}\right)^{2} \tau_{\mathrm{m}}+\left(1-\rho_{\mathrm{m}-\infty}\right)^{2} \rho_{\mathrm{m}-\infty} \tau_{\mathrm{m}}^{3} \rho_{\mathrm{app}, \mathrm{m}-\infty, 1}+\left(1-\rho_{\mathrm{m}-\infty}\right)^{2} \rho_{\mathrm{m}-\infty}^{2} \tau_{\mathrm{m}}^{5} \rho_{\mathrm{app}, \mathrm{m}-\infty, 1}^{2}+\ldots \\ = & \frac{\left(1-\rho_{\mathrm{m}-\infty}\right)^{2} \tau_{\mathrm{m}}}{1-\rho_{\mathrm{m}-\infty} \rho_{\mathrm{app}, \mathrm{m}-\infty, 1} \tau_{\mathrm{m}}^{2}}\end{aligned}$

Figure A-3. Radiation balance equation and coefficients used for the single-layer validation test

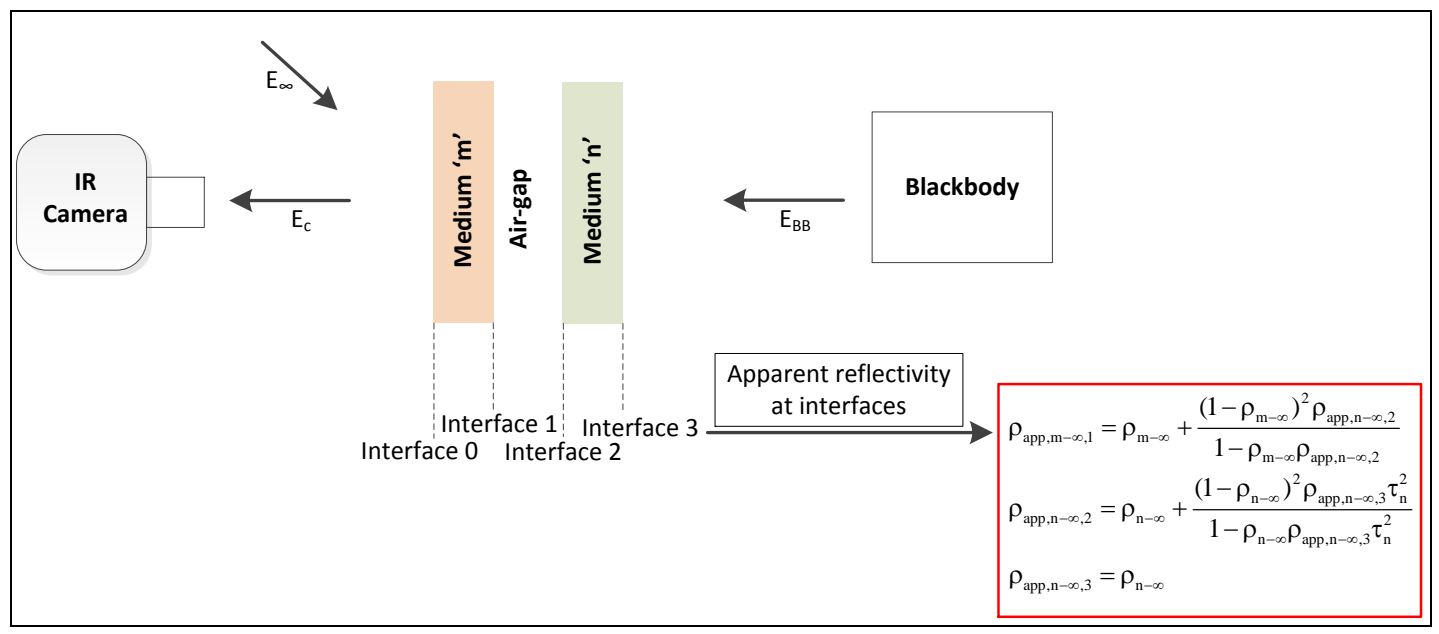

Figure A-4. Validation test with double layers of medium 


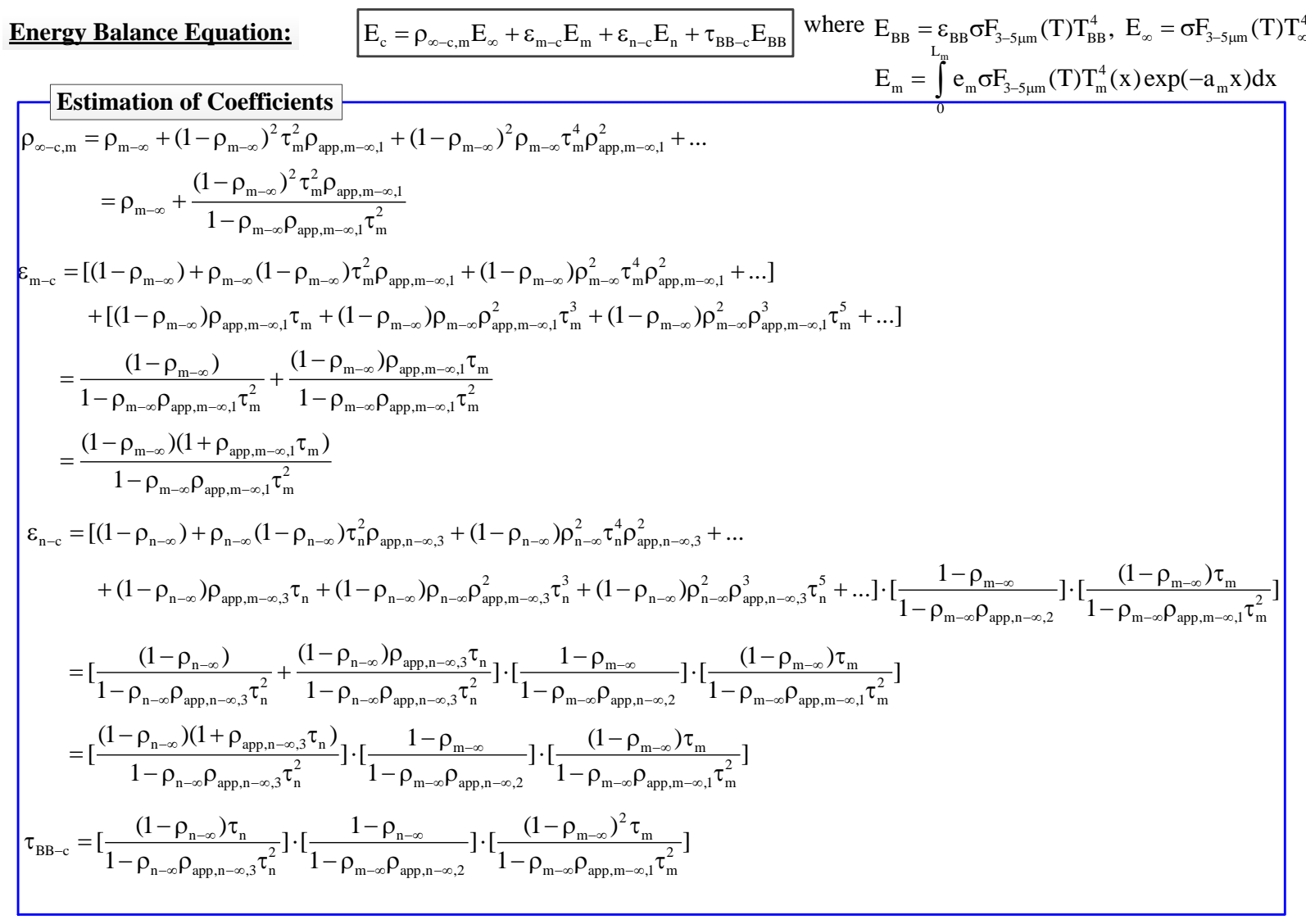

Figure A-5. Radiation balance equation and coefficients used for the double-layer validation test 
A.3. Heater wall temperature tracking through the multilayer

The wall temperature tracking through the multilayer shown in Figure 2 is performed using the two-way coupled algorithm between the radiation balance equation and 1D heat conduction equation (Section 3). In Figure A6 , the coupling algorithm and the governing equations employed are presented. It is noted that we defined the apparent reflectivities at interface 1 and interface 2 shown in Figure A- 6 to make the derivation of coefficients used in Eq. (1) more convenient. Using the definitions, the infinite number of transmissions/reflections between interface 1 and interface 3, between interface 2 and interface 3 can be treated implicitly during the derivation as shown in Figure A-7 which describes the derivation process of the coefficients for Eq. (1) with their physical meanings.

Also, to solve the Eq. (1), the amount of radiation emitted by each layer is estimated as follows:

First, for the semi-transparent layer to mid-wave IR radiation (i.e., sapphire, soda-lime glass), the volumetric effect of radiation must be considered to calculate the amount of energy emitted. In other words, $E_{\text {sap }}$ or $E_{g}$ is calculated by integrating the local emission and attenuation within the volume of each medium. Let us consider the radiation flux $\left(\mathrm{W} / \mathrm{m}^{2}\right)$ emitted by a differential layer of thickness $d x$ in the $x$-direction with an emission coefficient of $e_{m}\left(\mathrm{~m}^{-1}\right)$. Then, the radiation flux from the small thickness is given by $e_{m} \sigma F_{3-5 \mu m}(T) T_{m}^{4}(x) d x$. Considering the attenuation across the medium of thickness $L_{m}$, the total emission $\left(E_{m}\right)$ by a medium is obtained by

$$
\mathrm{E}_{\mathrm{m}}=\int_{0}^{\mathrm{L}_{\mathrm{m}}} \mathrm{e}_{\mathrm{m}} \sigma \mathrm{F}_{3-5 \mu \mathrm{m}}(\mathrm{T}) \mathrm{T}_{\mathrm{m}}^{4}(\mathrm{x}) \exp \left(-\mathrm{a}_{\mathrm{m}} \mathrm{x}\right) \mathrm{dx}
$$

where $m$ refers to the medium (i.e., sap or $g$ ), $e$ is the emission coefficient, $a$ is the absorption coefficient, $L_{m}$ is the thickness of the medium, and $T_{m}$ is the temperature of the medium.

Applying Kirchhoff's law, which assumes a relationship of equality between the emission and absorption coefficient (i.e., $e=a$ ), Eq. (2) can be rewritten as

$$
\mathrm{E}_{\mathrm{m}}=\int_{0}^{\mathrm{L}_{\mathrm{m}}} \mathrm{a}_{\mathrm{m}} \sigma \mathrm{F}_{3-5 \mu \mathrm{m}}(\mathrm{T}) \mathrm{T}_{\mathrm{m}}^{4}(\mathrm{x}) \exp \left(-\mathrm{a}_{\mathrm{m}} \mathrm{x}\right) \mathrm{dx}
$$

The radiation generated by the ITO film, which is opaque to IR radiation, is estimated as

$$
\mathrm{E}_{\text {гто }}=\varepsilon_{\text {гто }} \sigma \mathrm{F}_{3-5 \mu \mathrm{m}}(\mathrm{T}) \mathrm{T}_{\text {гто }}^{4}
$$

where $\varepsilon_{I T O}$ is the emissivity of ITO and $T_{\text {ITO }}$ is the temperature of the ITO film. 


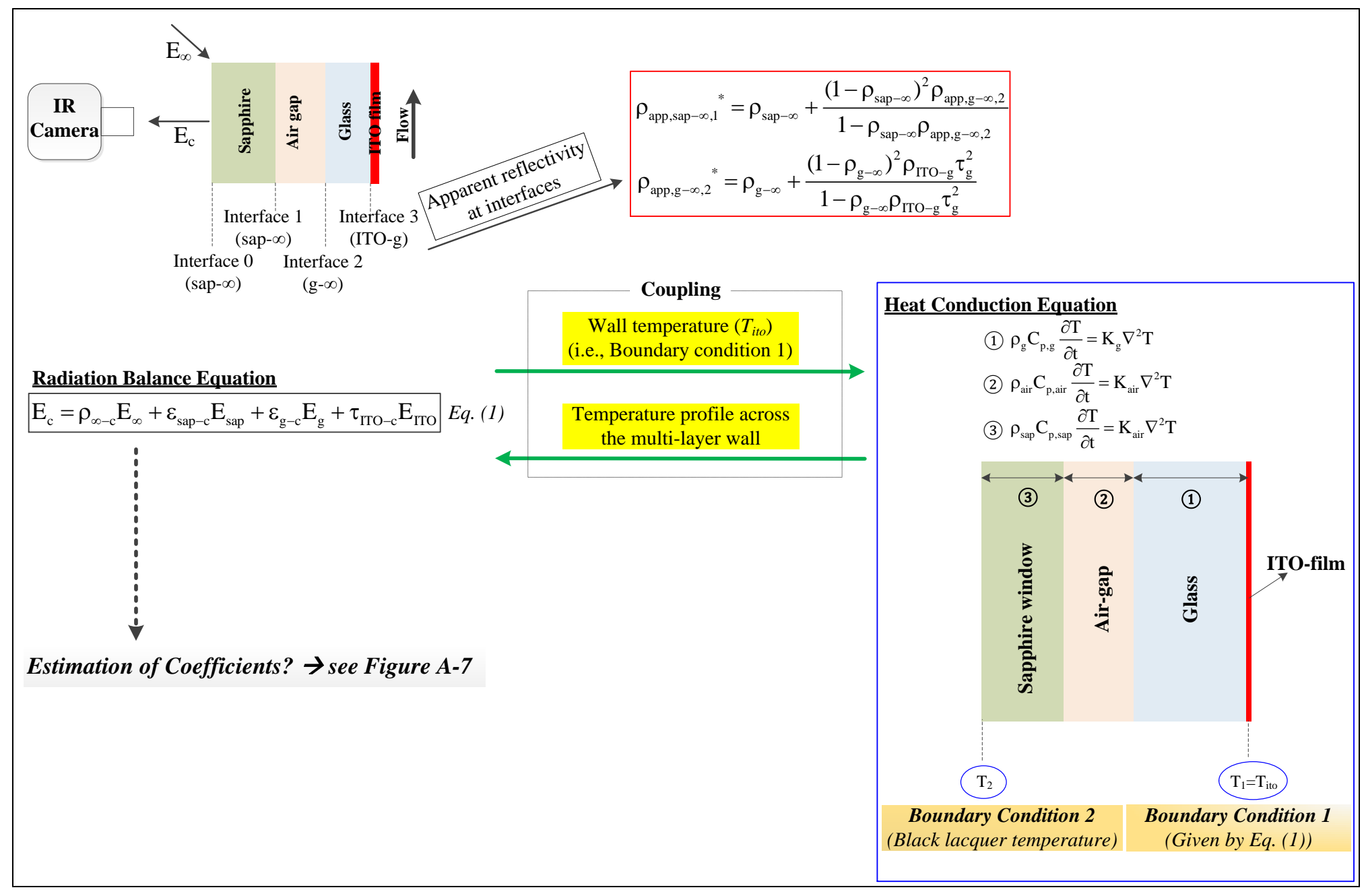

Figure A-6. Schematic of wall temperature tracking algorithm 


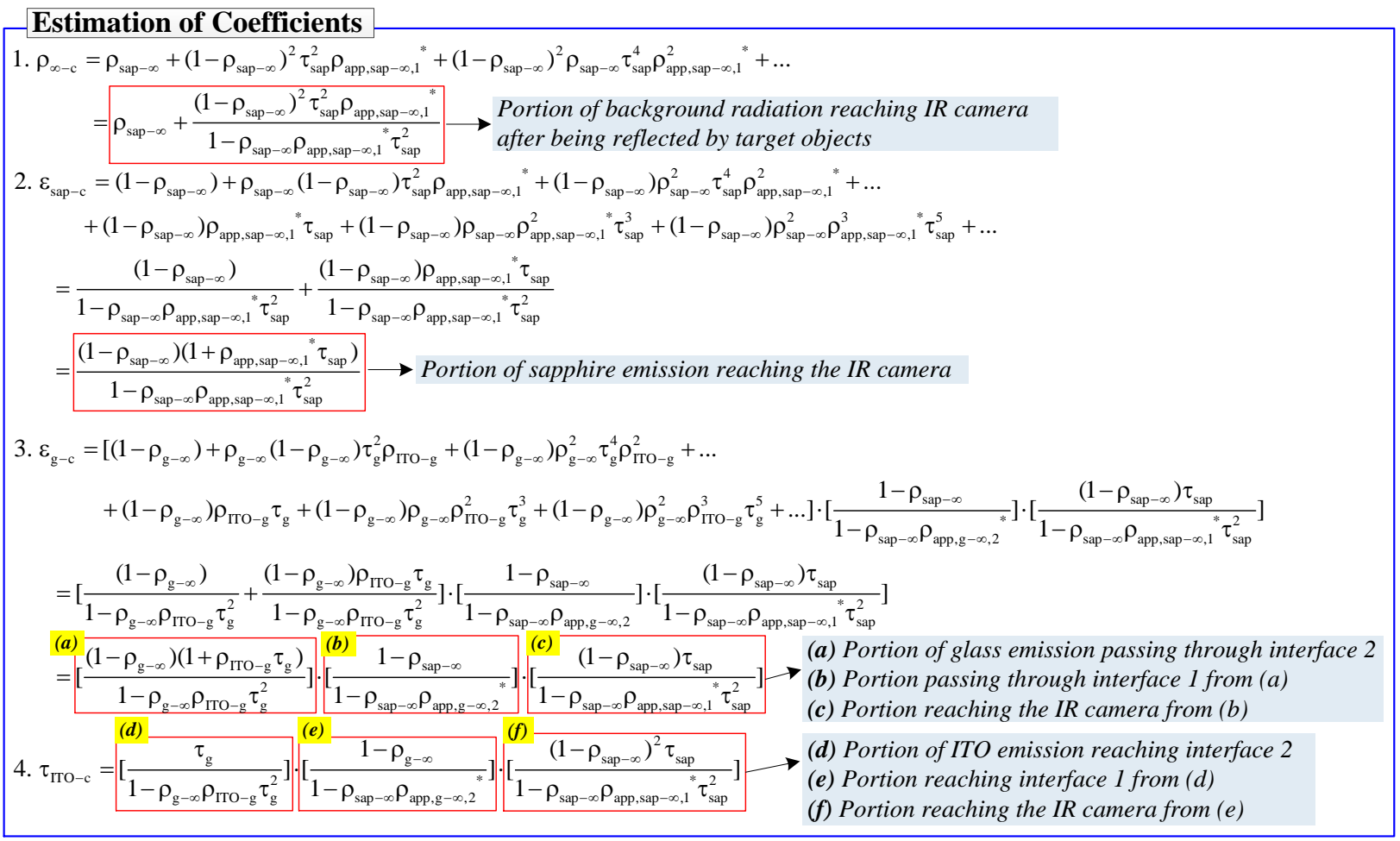

Figure A-7. Derivation of coefficients used in Eq. (1) 